\title{
BONIFICACIÓN DE DETERMINADAS CUOTAS DE LA SEGURIDAD SOCIAL EN MELILLA: EVALUACIONES Y PERSPECTIVAS
}

JESÚS A. GARCÍA AYALA

\begin{abstract}
Doctor en Ciencia Política y de la Administración por la Universidad de Granada Licenciado en Ciencias Económicas y Empresariales por la Universidad Complutense de Madrid
\end{abstract}

Resumen: El rechazo por el Congreso de los Diputados a la toma en consideración de la proposición de ley presentada por la Asamblea de la ciudad de Melilla, para la extensión y ampliación de las bonificaciones en determinadas cuotas de la Seguridad Social, puede haber trasladado el debate a la sociedad civil. A los actores políticos se sumarían ahora las centrales sindicales y organizaciones empresariales.

La reflexión a realizar entre todos se ve dificultada por una de las carencias puestas de manifiesto: la de datos objetivos y fiables sobre Melilla que permitan evaluar los resultados de las bonificaciones vigentes.

Por ello se hacen necesarios varios objetivos: el inicial es aportar datos válidos para la citada evaluación, el intermedio es que el tratamiento de los datos obtenidos permita la obtención de resultados representativos, y el final es la extracción de conclusiones y recomendaciones válidas.

Las conclusiones, asociadas a hechos objetivos tratados en el curso del trabajo, tratan de anticipar, en lo posible, las consecuencias que se seguirían en el supuesto de que las bonificaciones fueran extendidas y ampliadas, siguiendo la senda de convergencia deducible del debate parlamentario.

Finalmente se aportan unas determinadas consideraciones, cuyo carácter subjetivo no puede descartarse en tanto están asociadas a la opinión personal del autor.

Palabras clave: Melilla, cuotas a la Seguridad Social, bonificaciones a la Seguridad Social, bonificaciones en las cuotas, evaluación de bonificaciones, impacto económico y social, creación de empleo.

Clasificación JEL: E24, H24, H25, H55, J08, J23, J38, R11.

Abstract: The rejection by the Congress of the Deputies to the taking in consideration of the proposal of law, presented by the Assembly of the City of Melilla, for the extension and increase of the subsidies in certain contributions to Social Security, can have transferred the debate to the civil society. To the political actors, the unions and enterprise organizations would be added now. 
The reflection to make between everybody is made difficult by one of the shown deficiencies: the one of objective and trustworthy data on Melilla that allows to evaluate the results of the effective subsidies. For that reason several targets become necessary: the initial one is to contribute valid data for the mentioned evaluation; the intermediate one is that the treatment of the collected data allows the obtaining of representative results; and the next one is the extraction of valid conclusions and recommendations.

The conclusions, associated to objective facts treated in the course of the work, try to anticipate, as far as possible, the consequences that would be followed supposing that the subsidies were extended and increased, following the footpath of deductible convergence of the parliamentary debate.

Finally certain considerations are contributed, whose subjective character cannot discard in as much are associate to the personal opinion of the author.

Key words: Melilla, contributions to Social Security, subsidies to Social Security, subsidies in the contribution, evaluation of subsidies, economic and social impact, creation of employment.

\section{INTRODUCCIÓN}

La disposición adicional trigésima del texto refundido de la Ley General de la Seguridad Social, establece en su apartado dos, ${ }^{1}$ que los empresarios dedicados a actividades encuadradas en los Sectores de Comercio, Hostelería, Turismo e Industria, excepto Energía y Agua, respecto de los trabajadores que les presten servicios en sus centros de trabajo ubicados en las Ciudades de Ceuta y Melilla, tendrán derecho a una bonificación de hasta el 40 por 100 en sus aportaciones a las cuotas de la Seguridad Social por contingencias comunes, así como por los conceptos de recaudación conjunta con las mismas. Tal beneficio en la cotización y hasta ese porcentaje máximo se extiende también por dicho precepto legal a los trabajadores por cuenta propia de los Sectores antes citados, encuadrados en el Régimen Especial de Autónomos y que residan y ejerzan también su actividad en las Ciudades de Ceuta y Melilla, respecto de sus cuotas a la Seguridad Social por contingencias comunes. $^{2}$

${ }^{1}$ Conforme a la redacción dada al mismo por la disposición adicional 39a de la Ley 62/2003, de 30 de diciembre, de Medidas Fiscales, Administrativas y del Orden Social.

${ }^{2}$ La aplicación de estas bonificaciones se reguló mediante la ORDEN TAS/471/2004. Posteriormente, su aplicación se ha ido prorrogando cada dos años mediante las normas (que modificaron parcialmente la anterior) siguientes: ORDENTAS/585/2006, ORDENTAS/710/2008Y ORDENTIN/530/2010. Todas ellas se relacionan en el capítulo de NOTAS. 


\section{Modificación propuesta por la Asamblea de Melilla:}

La Asamblea de la Ciudad Autónoma de Melilla consideró que el sistema citado, aprobado en 2003 y prorrogado en varias ocasiones, pese a sus efectos positivos no era suficiente y debía ser mejorado para corregir los desequilibrios que se producen en dicha ciudad, derivados fundamentalmente de su condición extra peninsular, de lo limitado de su ámbito territorial y de la escasez de recursos naturales. Por todo ello, aprobó una iniciativa que pretendía ampliar del 40\% al 50\%, y extender a todos los sectores en crisis, las bonificaciones en las cuotas a la Seguridad Social que se aplican en Melilla, hasta ahora limitadas a los sectores del comercio, hostelería, turismo y a algunas ramas de la industria (las exceptuadas son energía y agua). El 19 de diciembre de 2007 se presentó en el registro del Congreso de los Diputados la iniciativa de la Asamblea de Melilla, consistente en una proposición de ley para la modificación del apartado 2 de la disposición adicional 30. ${ }^{a}$ del Real Decreto 1/1994, de 20 de junio, por el que se aprobó el texto refundido de la Ley General de la Seguridad Social, modificado en su día por la Ley 62/2003, de 30 de diciembre.

\section{Debate parlamentario, intervenciones y resultado:}

El 11 de mayo de 2010, en sesión plenaria del Congreso de los Diputados, se produjo el debate para la toma en consideración o el rechazo de dicha iniciativa.

La justificación a destacar por el representante de la Asamblea de Melilla, es que la actual regulación deja fuera de su ámbito de aplicación fundamentalmente a numerosas pequeñas empresas que no estaban encuadradas en los grupos originalmente beneficiados por la medida. [Se trata de] pequeñas empresas del sector servicios que no pueden beneficiarse ni siquiera del 40\%. De ahí la propuesta que se hace de extender la medida a todos los sectores económicos en crisis. Defiende además la elevación de la cuantía de la bonificación del 40 al 50\%, así como que la actual vigencia de dos años, prorrogables en su caso, pase a los cinco años, prorrogables asimismo en su caso. La aprobación de la iniciativa supondría un importante impulso a la economía de Melilla y a la generación de empleo [...], en una época de crisis global [...], en una ciudad que tiene una de las tasas de desempleo más altas en [...] nuestro país.

En cuanto a las posiciones de los representantes de los grupos parlamentarios ${ }^{3}$ que concluyeron sus intervenciones anunciando su rechazo ${ }^{4}$ o abstención, ${ }^{5}$ cabe destacar: a)

\footnotetext{
${ }^{3}$ Anunciaron su voto a favor: Grupo Parlamentario Mixto (Coalición Canaria), Grupo Parlamentario de Esquerra Republicana-Izquierda Unida-Iniciativa per Catalunya Verds, y Grupo Parlamentario Popular.

${ }^{4}$ Grupo Parlamentario Socialista.

${ }^{5}$ Grupo Parlamentario Vasco (EAJ-PNV), y Grupo Parlamentario Catalán (Convergència i Unió)
} 
Las críticas a la extensión de las bonificaciones a todos los sectores, así como a su generalidad y a su carácter estructural, y (menos radicales que las anteriores) a la extensión del 40\% al $50 \% ; b$ ) La convicción de que el instrumento normativo más apropiado son los Presupuestos Generales del Estado; c) El desconocimiento del impacto económico de las medidas, así como de posibles evaluaciones por parte de la Agencia de Evaluación de Políticas Públicas, la Universidad o los gobiernos autonómicos afectados; d) La convicción de que en cualquier caso la iniciativa debe evaluarse antes por el Comité de Seguimiento del Pacto de Toledo; e) Una experiencia empírica genérica que no avala la consecución por este tipo de medidas de los efectos que persiguen; $f$ ) En definitiva, las reservas a la eficiencia de las propuestas contenidas en la iniciativa (bonificaciones) con relación a sus objetivos (empleo).

El resultado de la votación sobre la toma en consideración de la proposición de ley referida fue el siguiente: votos emitidos, 332; a favor, 146; en contra, 167; abstenciones, 19. La iniciativa quedó por tanto rechazada.

\section{Literatura invocada en apoyo de las opiniones manifestadas:}

La literatura científica invocada expresamente por los diferentes grupos parlamentarios intervinientes en el debate, en materia de eficiencia de las actuaciones propuestas (bonificaciones) con relación a los objetivos perseguidos (creación de empleo) se indica a continuación.

Por parte del Grupo Vasco (EAJ-PNV) ${ }^{6}$ se invocan, en primer lugar, las comparecencias y los estudios y evaluaciones realizados en la Comisión de seguimiento del Pacto de Toledo: determinarían que la bonificación de contingencias o de cuotas a la Seguridad Social no es el mejor instrumento para la creación de empleo y que los hay mejores. En segundo lugar, se invoca la comprobación empírica mediante las aportaciones doctrinales del estudio del profesor Toharia y otros: revelaría que las bonificaciones de cuotas son un instrumento neutro o ineficiente en la generación de empleo.

Por el Grupo Catalán (Convergència i Unió) ${ }^{7}$ se invoca a la Agencia de Evaluación de Políticas Públicas y un informe de la Universidad de Alcalá: ambos indicarían que la política española de bonificaciones, caracterizada por una amplitud excesiva, no ha tenido efectos significativos en la creación de empleo.

\footnotetext{
${ }^{6}$ Señor-OLABARRÍA MUÑOZ.

${ }^{7}$ Señor-CAMPUZANO I CANADÉS.
} 
Por su parte, el Grupo de Esquerra Republicana-Izquierda Unida-Iniciativa per Catalunya Verds ${ }^{8}$ afirma estar convencido de que no existen estudios comparados que demuestren que las bonificaciones en las cuotas sociales efectúan una mínima aportación a la competitividad empresarial y a la mejora económica.

En cuanto al Grupo Socialista, ${ }^{9}$ recuerda las largas horas de debate reciente sobre qué hacer con las bonificaciones a la Seguridad Social, y la subsiguiente petición de informes sobre su supuesto impacto en la creación de empleo.

\section{Importancia futura:}

La presidencia de la ciudad de Melilla ha manifestado la intención de volver a presentar en un futuro próximo una iniciativa similar a la rechazada, previa su adaptación a lo manifestado en el debate por los grupos parlamentarios distintos al popular. Por otra parte, también la presidencia de la ciudad de Ceuta manifiesta su voluntad de presentar una iniciativa análoga, en un momento todavía pendiente de determinar. De ambas intenciones surge la importancia de estudiar las manifestaciones referidas, al objeto de que los resultados que se obtengan puedan ser utilizados, en su caso, en la configuración de las iniciativas anunciadas.

\section{Precisión de los objetivos del presente trabajo:}

Dentro del marco de los objetivos generales a que se ha hecho referencia en el resumen, los objetivos instrumentales que se persiguen pueden sintetizarse en los cuatro que se indican a continuación.

Primero, una aproximación al impacto económico y social de las medidas propuestas y rechazadas, incluida la cuantificación del coste de las mismas para la hacienda pública, en términos de lo que esta dejaría de recaudar.

Segundo, la detección y estudio de las evaluaciones oficiales que puedan existir para el caso de Melilla (y de Ceuta), desconocidas o insuficientemente conocidas para quienes intervinieron en el debate parlamentario.

Tercero, a partir de las posibles evaluaciones oficiales, la aportación añadida propia a la evaluación de las diferentes variables reglamentarias: afiliación a la Seguridad Social, bonificaciones, contrataciones, tasas de actividad, tasas de empleo y paro.

\footnotetext{
${ }^{8}$ Señor LLAMAZARES TRIGO.

${ }^{9}$ Señora LÓPEZ I CHAMOSA.
} 
Cuarto, la evaluación propia de otras variables concernidas: convenios colectivos, remuneraciones por asalariado, contratación indefinida, PIB por habitante y VAB por sectores.

Todos los objetivos intentan abordarse dentro de una metodología científica, de carácter empírico o prospectivo, según los casos, y siempre en el marco de la naturaleza objetiva, independiente y reproducible caracterizadora de la investigación científica.

\section{APROXIMACIÓN AL IMPACTO ECONÓMICO DE LAS MEDIDAS}

Esta aproximación parte de la posterior tabla de síntesis (1.1) elaborada para cuantificar las repercusiones directas en los distintos números de trabajadores afectados, y sus importes correspondientes, de las bonificaciones inicialmente propuestas y finalmente rechazadas. El período base de referencia ha sido marzo de 2010.

Como puede comprobarse, los trabajadores cuyas cuotas son bonificadas habrían aumentado en 7.909 (988 de ellos autónomos), pasando de 5.867 a 13.776. Por su parte, el importe mensual habría aumentado en 1.493.118 $€$, al pasar de $751.888 €$ a 2.245.006 $€$. En cómputo anual el importe habría aumentado en 17.917 .414 ", al pasar de $9.022 .656 €$ a $26.940 .070 €$. Sobre tal base, el importe total para los cuatro años propuestos se habría situado en 107.760.279 €.

Tabla 1.1: Impacto económico de las medidas

MELILLA: BONIFICACIONES DE CUOTAS A LA SEGURIDAD SOCIAL

\begin{tabular}{|c|c|c|}
\hline I. BONIFICACIÓN RÉGIMEN GENERAL & & 31-mar-2010 \\
\hline TRABAJADORES EN ALTA & (a) & 15.328 \\
\hline TRABAJADORES CON BONIFICACIÓN & (b) & 3.715 \\
\hline TRABAJADORES SIN BONIFICACIÓN & $(c)=(a)-(b)$ & 11.613 \\
\hline TRABAJADORES SECTOR PÚBLICO & (d) & 6.119 \\
\hline RESTO TRABAJADORES BONIFICABLES & $(e)=(c)-(d)$ & 5.494 \\
\hline TOTAL TRABAJADORES BONIFICABLES & $(f)=(b)+(e)$ & 9.209 \\
\hline IMPORTE BONIFICADO (40\%) TOTAL/MES & (g) & $510.786,00 €$ \\
\hline IMPORTE BONIFICADO (40\%) TOTAL/AÑO & $(\mathrm{h})=(\mathrm{g}) \times 12$ & $6.129 .432,00 €$ \\
\hline IMPORTE BONIFICADO (40\%) UNITARIO/MES & $(\mathrm{i})=(\mathrm{g}) /(\mathrm{b})$ & $137,49 €$ \\
\hline IMPORTE BONIFICADO (50\%) UNITARIO/MES & $(\mathrm{j})=(\mathrm{i}) / 4 \times 5$ & $171,87 €$ \\
\hline IMPORTE BONIFICABLE $(50 \%)$ TOTAL/MES & $(\mathrm{k})=(\mathrm{f}) \mathrm{x}(\mathrm{j})$ & $1.582 .714,76 €$ \\
\hline IMPORTE BONIFICABLE DIFERENCIAL/MES & $(\mathrm{m})=(\mathrm{k})-(\mathrm{g})$ & $1.071 .928,76 €$ \\
\hline IMPORTE BONIFICABLE (50\%) TOTAL/AÑO & $(\mathrm{n})=(\mathrm{k}) \times 12$ & $18.992 .577,15 €$ \\
\hline IMPORTE BONIFICABLE DIFERENCIAL (AÑO) & $(\tilde{\mathrm{n}})=(\mathrm{n})-(\mathrm{h})$ & 12.863.145,15 € \\
\hline
\end{tabular}




\begin{tabular}{|c|c|c|}
\hline II. BONIFICACIÓN RÉGIMEN ESPECIAL AUTÓNOMOS & & 31-mar-2010 \\
\hline TRABAJADORES EN ALTA & (a) & 3.140 \\
\hline TRABAJADORES CON BONIFICACIÓN & (b) & 2.152 \\
\hline TRABAJADORES SIN BONIFICACIÓN & $(c)=(a)-(b)$ & 988 \\
\hline TRABAJADORES SECTOR PÚBLICO & (d) & 0 \\
\hline RESTO TRABAJADORES BONIFICABLES & $(\mathrm{e})=(\mathrm{c})-(\mathrm{d})$ & 988 \\
\hline TOTAL TRABAJADORES BONIFICABLES & $(f)=(b)+(e)$ & 3.140 \\
\hline IMPORTE BONIFICADO $(40 \%)$ TOTAL/MES & (g) & $241.102,00 €$ \\
\hline IMPORTE BONIFICADO (40\%) TOTAL/AÑO & $(\mathrm{h})=(\mathrm{g}) \times 12$ & $2.893 .224,00 €$ \\
\hline IMPORTE BONIFICADO (40\%) UNITARIO/MES & $(\mathrm{i})=(\mathrm{g}) /(\mathrm{b})$ & $112,04 €$ \\
\hline IMPORTE BONIFICADO $(50 \%)$ UNITARIO/MES & $(\mathrm{j})=(\mathrm{i}) / 4 \times 5$ & $140,05 €$ \\
\hline IMPORTE BONIFICABLE $(50 \%)$ TOTAL/MES & $(\mathrm{k})=(\mathrm{f}) \mathrm{x}(\mathrm{j})$ & $439.742,26 €$ \\
\hline IMPORTE BONIFICABLE DIFERENCIAL/MES & $(\mathrm{m})=(\mathrm{k})-(\mathrm{g})$ & $198.640,26 €$ \\
\hline IMPORTE BONIFICABLE (50\%) TOTAL/AÑO & $(\mathrm{n})=(\mathrm{k}) \times 12$ & $5.276 .907,16 €$ \\
\hline IMPORTE BONIFICABLE DIFERENCIAL (AÑO) & $(\tilde{\mathrm{n}})=(\mathrm{n})-(\mathrm{h})$ & 2.383.683,16€ \\
\hline
\end{tabular}

\begin{tabular}{|c|c|c|}
\hline \multicolumn{2}{|c|}{$\begin{array}{l}\text { III. BONIFICACIÓN REGÍMENES ESPECIALES AGRARIO, HOGAR, MAR Y } \\
\text { CARBÓN }\end{array}$} & \multirow{2}{*}{$\begin{array}{r}\text { 31-mar-2010 } \\
1.427\end{array}$} \\
\hline $\begin{array}{r}\text { TRABAJADORES EN ALTA } \\
\end{array}$ & (a) & \\
\hline TRABAJADORES CON BONIFICACIÓN & (b) & 0 \\
\hline TRABAJADORES SIN BONIFICACIÓN & $(\mathrm{c})=(\mathrm{a})-(\mathrm{b})$ & 1.427 \\
\hline TRABAJADORES SECTOR PÚBLICO & (d) & 0 \\
\hline RESTO TRABAJADORES BONIFICABLES & $(e)=(c)-(d)$ & 1.427 \\
\hline TOTAL TRABAJADORES BONIFICABLES & $(f)=(b)+(e)$ & 1.427 \\
\hline IMPORTE BONIFICADO (40\%) TOTAL/MES & (g) & $-€$ \\
\hline IMPORTE BONIFICADO (40\%) TOTAL/AÑO & $(\mathrm{h})=(\mathrm{g}) \times 12$ & $-€$ \\
\hline IMPORTE BONIFICADO (40\%) UNITARIO/MES & $(\mathrm{i})=(\mathrm{g}) /(\mathrm{b})$ & $-€$ \\
\hline IMPORTE BONIFICADO (50\%) UNITARIO/MES & $(\mathrm{j})=(\mathrm{i}) / 4 \times 5$ & $155,96 €$ \\
\hline IMPORTE BONIFICABLE (50\%) TOTAL/MES & $(\mathrm{k})=(\mathrm{f}) \mathrm{x}(\mathrm{j})$ & $222.548,78 €$ \\
\hline IMPORTE BONIFICABLE DIFERENCIAL/MES & $(\mathrm{m})=(\mathrm{k})-(\mathrm{g})$ & $222.548,78 €$ \\
\hline IMPORTE BONIFICABLE $(50 \%)$ TOTAL/AÑO & $(\mathrm{n})=(\mathrm{k}) \mathrm{x} 12$ & $2.670 .585,32 €$ \\
\hline IMPORTE BONIFICABLE DIFERENCIAL (AÑO) & $(\tilde{\mathrm{n}})=(\mathrm{n})-(\mathrm{h})$ & $2.670 .585,32 €$ \\
\hline
\end{tabular}

\begin{tabular}{|c|c|c|}
\hline IV. TOTAL BONIFICACIÓN REGÍMENES GRAL. Y ESPECIALES & & 31-mar-2010 \\
\hline TRABAJADORES EN ALTA & (a) & 19.895 \\
\hline TRABAJADORES CON BONIFICACIÓN & (b) & 5.867 \\
\hline TRABAJADORES SIN BONIFICACIÓN & $(c)=(a)-(b)$ & 14.028 \\
\hline TRABAJADORES SECTOR PÚBLICO & (d) & 6.119 \\
\hline RESTO TRABAJADORES BONIFICABLES & $(e)=(c)-(d)$ & 7.909 \\
\hline TOTAL TRABAJADORES BONIFICABLES & $(f)=(b)+(e)$ & 13.776 \\
\hline IMPORTE BONIFICADO $(40 \%)$ TOTAL/MES & $(\mathrm{g})$ & $751.888,00 €$ \\
\hline IMPORTE BONIFICADO (40\%) TOTAL/AÑO & $(\mathrm{h})=(\mathrm{g}) \times 12$ & $9.022 .656,00 €$ \\
\hline IMPORTE BONIFICADO (40\%) UNITARIO/MES & $(\mathrm{i})=(\mathrm{g}) /(\mathrm{b})$ & $249,53 €$ \\
\hline IMPORTE BONIFICADO (50\%) UNITARIO/MES & $(j)=(i) / 4 \times 5$ & $467,87 €$ \\
\hline IMPORTE BONIFICABLE (50\%) TOTAL/MES & $(\mathrm{k})=(\mathrm{f}) \mathrm{x}(\mathrm{j})$ & $2.245 .005,80 €$ \\
\hline IMPORTE BONIFICABLE DIFERENCIAL/MES & $(\mathrm{m})=(\mathrm{k})-(\mathrm{g})$ & $1.493 .117,80 €$ \\
\hline IMPORTE BONIFICABLE (50\%) TOTAL/AÑO & $(\mathrm{n})=(\mathrm{k}) \mathrm{x} 12$ & $26.940 .069,63 €$ \\
\hline IMPORTE BONIFICABLE DIFERENCIAL (AÑO) & $(\tilde{\mathrm{n}})=(\mathrm{n})-(\mathrm{h})$ & $17.917 .413,63 €$ \\
\hline
\end{tabular}

FUENTE: Elaboración propia a partir de datos oficiales de la Tesorería General de la Seguridad Social (marzo 2010): <http://www.seg-social.es> 
Por citar alguna comparación, los 17.917.414 “ anuales (en importes de 2009) en que se cuantifica económicamente la repercusión empresarial directa del rechazo del Congreso de los Diputados a la iniciativa de la Asamblea de la ciudad de Melilla, vienen a representar el 58'6\% de las inversiones públicas contenidas para Melilla en los Presupuestos Generales del Estado para dicho año, y el 171'2\% de los Fondos de Compensación Interterritoral (Fondo de Compensación y Fondo Complementario) asignados a Melilla en los mismos presupuestos. Y ello sin computar los efectos indirectos, tanto en términos de actividad económica de la ciudad de Melilla, como de efectos sociales a favor de los trabajadores, consumidores y usuarios en la misma.

\section{EVALUACIONES REGLAMENTARIAS OFICIALES}

La antes citada disposición adicional trigésima segunda preceptúa, como se ha dicho, que las referidas bonificaciones se establecerán, en su caso, por un período de tiempo limitado, a los efectos de proceder a evaluar periódicamente el grado de eficacia de la misma en relación con los objetivos sociales que se pretenden alcanzar.

Tras sucesivas modificaciones de los términos en que debe desarrollarse la evaluación citada, lo vigente actualmente es lo establecido por la ORDENTIN/530/23010 (que en algunos aspectos se remite a las anteriores, antes relacionadas).

La tabla que se inserta posteriormente (2.1) sintetiza el marco reglamentario en el que deben desarrollarse actualmente las evaluaciones, incluidos objetivos, indicadores y cumplimientos.

Por su parte, la Agencia Estatal de Evaluación de las Políticas Públicas y la Calidad de los Servicios efectuó una evaluación, referida a 2004, 2005 y 2006, sobre "La bonificación de las cuotas sociales a la Seguridad Social en Ceuta y Melilla para estimular la creación de empleo". Sus principales conclusiones y recomendaciones se sintetizan a continuación.

- Conclusiones:

a) En cuanto a afiliación a la Seguridad Social, se ha registrado "una situación positiva [...] en los sectores bonificados [...] que puede interpretarse como un funcionamiento adecuado de la medida";

b) En materia de contrataciones "el crecimiento [...] es más intenso que en el ámbito nacional, llegando incluso a duplicarlo";

c) En lo referente al coste de la política de bonificación, supera los "siete millones 
de euros anuales" y "el número de empleos afectados por la misma en promedio del período supera los [...] 1.850 autónomos y 3.400 empleados por cuenta ajena";

d) "El contenido más importante de estos acuerdos [...] es la obligación de las empresas beneficiarias de abonar un complemento retributivo [...] En el caso de Melilla [a los] Trabajadores con contrato indefinido, temporal a tiempo completo y parcial [...] Trabajadores con permiso de trabajo tipo "F" [...] [y] Trabajadores con contrato de formación [...] Estos acuerdos locales, al disminuir la bonificación efectiva que mantiene y estimula la creación de empleo, podrían estar limitando la consecución de los objetivos de la propia política, su eficacia”.

- Recomendaciones:

a) "Si el objetivo de la política de bonificación de las cuotas sociales es contribuir a la generación de empleo y el mantenimiento del existente, debería adecuarse su aplicación a la consecución estricta de este objetivo. La mejora de la renta disponible de las familias es otro objetivo diferente (al que seguramente contribuirá la consecución del primero), que no debería alterar la aplicación estricta de la política de bonificación de las cuotas sociales";

b) "Por motivos de eficiencia, se debería ampliar la política de bonificación de las cuotas sociales a todos los sectores de las economías de [...] Melilla. Si existen razones graves que justifiquen la exclusión de la medida de algún sector económico determinado, deberían enumerarse explícita y públicamente";

c) "Se podría variar la cuantía de la bonificación de las cuotas sociales para poder discriminar positivamente en proporción al número de trabajadores por cuenta ajena de la empresa beneficiaria”.

Es de destacar por otra parte la Recomendación n. ${ }^{\circ} 8$ del "Informe de la Ponencia para el análisis de los problemas estructurales del sistema de la Seguridad Social y de las principales reformas que deberán acometerse”, más conocido como «Pacto de Toledo», haciendo "suyas las recomendaciones contenidas en el «Libro Blanco de Delors» ${ }^{10}$ relativas a la reducción de las cotizaciones sociales, como elemento dinamizador del empleo [...]”.

${ }^{10}$ DELORS, J (1993). 


\section{Tabla 2.1: Marco reglamentario para las evaluaciones}

\begin{tabular}{|c|c|}
\hline \multicolumn{2}{|c|}{ OBJETIVOS (Orden TIN/530/2010) } \\
\hline PRECEPTO & Art. 1 (párrafo 1) Orden TAS/471/2004 según redacción Orden TAS/856/2006 \\
\hline TEXTO & $\begin{array}{l}\text { Procurar el mantenimiento y estimular la creación de empleo en Melilla, en concreto en los Sectores } \\
\text { de Comercio, Hostelería, Turismo y en la mayoría de las ramas de la Industria. }\end{array}$ \\
\hline \multicolumn{2}{|c|}{ INDICADORES (Orden TIN/530/2010) } \\
\hline PRECEPTO & Art. 1 (párrafo 2) Orden TAS/471/2004 según redacción Orden TIN/530/2010 \\
\hline \multirow[t]{8}{*}{ TEXTO } & Evolución de los datos en los sectores a los que va dirigida la medida: \\
\hline & - Afiliación a la Seguridad Social respecto de los trabajadores en alta. \\
\hline & - Bonificaciones. \\
\hline & - Contratación. \\
\hline & Datos referidos a Melilla: \\
\hline & - Tasas de actividad. \\
\hline & - Tasas de empleo. \\
\hline & - Paro. \\
\hline \multicolumn{2}{|c|}{ CUMPLIMIENTO DE OBJETIVOS (Orden TIN/530/2010) } \\
\hline PRECEPTO & Art. 1 (párrafo 2) Orden TAS/471/2004 según redacción Orden TIN/530/2010 \\
\hline TEXTO & $\begin{array}{l}\text { Cuando la evolución de los datos medidos por los indicadores converja en términos relativos con los } \\
\text { correspondientes datos agregados a nivel nacional. }\end{array}$ \\
\hline
\end{tabular}

FUENTE: Elaboración propia a partir del BOE núm. 59 de 9 de marzo de 2010 (Págs. 23637 y 23638)

\section{EVALUACIÓN PROPIA DE LAS VARIABLES REGLAMENTARIAS}

A continuación se procede a realizar una aproximación propia a la evaluación del sistema. Las limitaciones en que necesariamente va a moverse son fundamentalmente de orden estadístico, habida cuenta que no se dispone de datos oficiales con los grados de detalle precisos, es decir, de datos documentales de partida sobre, por una parte, España y Melilla (frente a datos conjuntos para Ceuta y Melilla); que, además, estén desglosados en las diferentes ramas de actividades concernidas: comercio, hostelería, turismo e industria (excepto energía y agua); y que, adicionalmente, se extiendan a todo el período a evaluar (enero 2004-abril 2010).

\subsection{Afiliación a la Seguridad Social}

En ausencia de datos sobre afiliaciones a la Seguridad Social en Melilla en los sectores 
bonificados, con respecto al total de trabajadores en alta, se han utilizado los datos agregados para Melilla y para el total de España. Son los recogidos en la tabla (3.1) y los gráficos (3.1 y 3.2) que se insertan seguidamente.

\section{Tabla 3.1:Trabajadores afiliados a la Seguridad Social}

\begin{tabular}{|c|c|c|c|c|c|c|}
\hline \multicolumn{7}{|c|}{$\begin{array}{l}\text { TRABAJADORES AFILIADOS A LA SEGURIDAD SOCIAL EN MELILLA Y } \\
\text { NUMERO ABSOLUTO DE PERSONAS Y \% DE INCREMENTOS }\end{array}$} \\
\hline \multirow[b]{3}{*}{ MES-AÑO } & \multicolumn{6}{|c|}{$\begin{array}{c}\text { TOTAL } \\
\end{array}$} \\
\hline & MELILLA & ESPAÑA & MELILLA & ESPAÑA & MELILLA & ESPAÑA \\
\hline & Personas & $\begin{array}{l}\text { Miles de } \\
\text { personas }\end{array}$ & 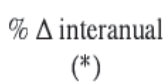 & $\begin{array}{c}\% \Delta \text { interanual } \\
(*)\end{array}$ & $\begin{array}{c}\% \Delta \\
\text { acumulado }\end{array}$ & $\begin{array}{c}\% \Delta \\
\text { acumulado }\end{array}$ \\
\hline $\begin{array}{l}\text { dic-04 } \\
\end{array}$ & 16.384 & 17.162 & - & - & - & - \\
\hline dic-05 & 17.207 & 18.156 & $5,0 \%$ & $5,8 \%$ & $5,0 \%$ & $5,8 \%$ \\
\hline dic-06 & 19.010 & 18.770 & $10,5 \%$ & $3,4 \%$ & $16,0 \%$ & $9,4 \%$ \\
\hline dic-07 & 19.391 & 19.196 & $2,0 \%$ & $2,3 \%$ & $18,4 \%$ & $11,9 \%$ \\
\hline dic-08 & 19.529 & 18.306 & $0,7 \%$ & $-4,6 \%$ & $19,2 \%$ & $6,7 \%$ \\
\hline dic-09 & 20.103 & 17.640 & $2,9 \%$ & $-3,6 \%$ & $22,7 \%$ & $2,8 \%$ \\
\hline abr-10 & 18.764 & 17.604 & $-6,7 \%$ & $-0,2 \%$ & $14,5 \%$ & $2,6 \%$ \\
\hline
\end{tabular}

(*) Excepto en el caso de abril 2010 (sobre diciembre 2009)

\section{Gráfico 3.1:Trabajadores afiliados}

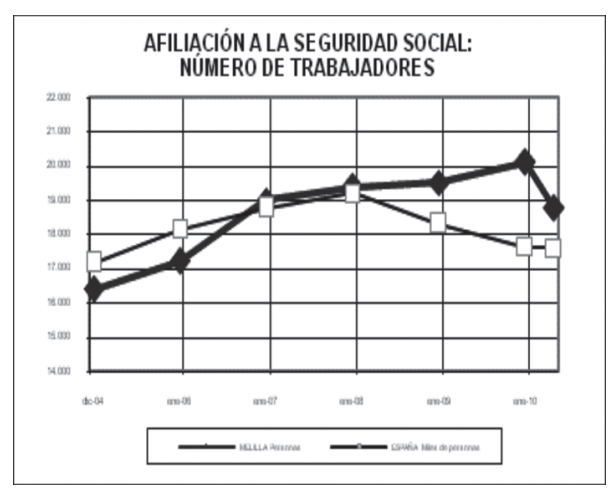

Gráfico 3.2: Incrementos de afiliados

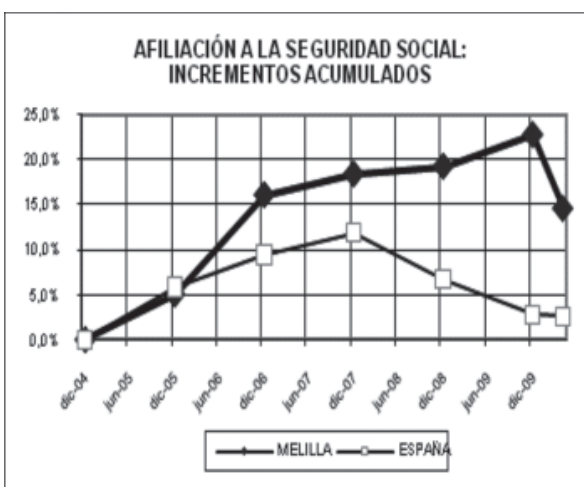

Fuente: Elaboración propia a partir de datos del Ministerio de Trabajo e Inmigración (2010)

Como puede observarse, tomando c $₫$ ßı año base 2004, el incremento acumulado de afiliaciones a la Seguridad Social hasta abril de 2010 ha sido superior en el caso de 
Melilla al caso del total de España, con las siguientes particularidades: el diferencial a favor de Melilla empezó a experimentarse a partir de diciembre de 2005, hasta llegar progresivamente a un máximo en diciembre de 2009 (22'7\% acumulado en Melilla frente al 2' $8 \%$ acumulado en el total España). En abril de 2010 el diferencial sigue siendo positivo para Melilla, si bien en una cuantía menor a la acabada de detallar (14'5\% acumulado en Melilla frente al 2'6\% acumulado en el total España). El descenso en 2010 de los efectos diferenciales citados puede indicar un agotamiento del potencial de la citada variable independiente: las bonificaciones.

Si bien no puede afirmarse que la bonificación de las cotizaciones sociales (supuesta variable independiente) en Melilla sea la única causante de la evolución diferencial positiva registrada por las afiliaciones totales (supuesta variable dependiente) en dicha ciudad, sí resulta acreditada la existencia de una determinada correlación positiva entre ambas variables; en efecto, el Coeficiente de Correlación ${ }^{11}$ para el período 2004-2010 $0^{12}$ resulta de 0'882271 (presenta a su vez un Coeficiente R2 de 0'778402). ${ }^{13}$

\subsection{Bonificaciones}

En la tabla (3.2) y los gráficos (3.3 y 3.4) siguientes'se aportan datos sobre las referidas bonificaciones en Melilla de cuotas a la Seguridad Social; se trata de datos agregados al no disponerse de su desglose por los sectores concernidos. Los desgloses de los que sí se ha dispuesto son los siguientes: a) Por trabajadores cuyas cuotas han sido

\footnotetext{
${ }^{11}$ La ecuación para el coeficiente de correlación es:

$o_{s, b}=\frac{\operatorname{Cov}(X, Y)}{\sigma_{s} \cdot \sigma_{H}}$
}

donde x e y son las medias de muestra PROMEDIO(matriz1) y PROMEDIO(matriz2).

Este coeficiente permite determinar la relación entre dos propiedades.

${ }^{12}$ Los importes utilizados en el cálculo son los que se recogen en el epígrafe siguiente (bonificaciones) y las afiliaciones las incluidas en este.

${ }^{13}$ La ecuación para el coeficiente de correlación del momento del producto Pearson, r, es:

$$
r=\frac{\sum(x-\bar{x})(y-\bar{y})}{\sqrt{\sum(x-\bar{x})^{2} \sum(y-\bar{y})^{2}}}
$$

donde $\mathrm{x}$ e y son las medias de muestra PROMEDIO(conocido_x) y PROMEDIO(conocido y).

Este coeficiente se puede interpretar como la proporción de la varianza de y, que puede atribuirse a la varianza de $\mathrm{x}$. 
objeto de bonificación; b) Por los importes correspondientes a dichos trabajadores; c) Por Régimen Especial de Autónomos y Régimen General; d) Por los años comprendidos, es decir, de 2004 a 2010, ambos inclusive (los datos para 2010 se han estimado para todo el año en proporción a los datos disponibles al término del primer trimestre).

\section{Tabla 3.2: Bonificaciones de cuotas}

\begin{tabular}{|c|c|c|c|c|c|c|c|c|}
\hline \multirow[b]{2}{*}{$\begin{array}{l}n \\
0 \\
0 \\
0 \\
0 \\
\text { 息 } \\
\text { 至 }\end{array}$} & \multicolumn{2}{|c|}{$\begin{array}{l}\text { RÉGIMEN ESPECIAL } \\
\text { AUTÓNOMOS(REA) }\end{array}$} & \multicolumn{3}{|c|}{ RÉGIMEN GENERAL (RG) } & \multicolumn{3}{|c|}{ TOTAL (T) } \\
\hline & 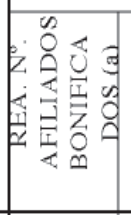 & 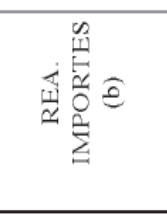 & 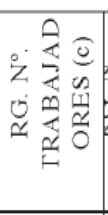 & 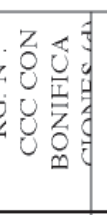 & 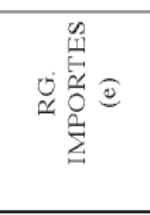 & 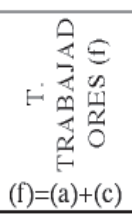 & 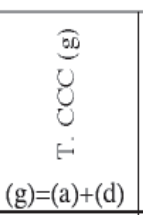 & 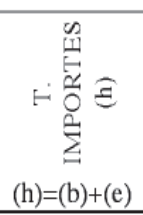 \\
\hline 2004 & 13.944 & $1.273 .407 €$ & & 8.236 & $2.782 .357 €$ & 13.944 & 22.180 & $4.055 .764 €$ \\
\hline 2005 & 17.937 & $1.685 .705 €$ & & 12.241 & $4.652 .132 €$ & 17.937 & 30.178 & $6.337 .837 €$ \\
\hline 2006 & 20.018 & $1.970 .262 €$ & 10.885 & 13.202 & $5.152 .086 €$ & 30.903 & 33.220 & $7.122 .348 €$ \\
\hline 2007 & 22.451 & $2.296 .580 €$ & 44.614 & 13.240 & $5.555 .217 €$ & 67.065 & 35.691 & $7.851 .797 €$ \\
\hline 2008 & 24.824 & $2.606 .767 €$ & 46.281 & 13.545 & $5.940 .740 €$ & 71.105 & 38.369 & $8.547 .507 €$ \\
\hline 2009 & 25.189 & $2.744 .373 €$ & 44.707 & 13.546 & $5.910 .927 €$ & 69.896 & 38.735 & $8.655 .300 €$ \\
\hline $\begin{array}{l}\text { SUBTOTAL } \\
\text { 2004-2009 }\end{array}$ & 124.363 & $12.577 .094 €$ & 146.487 & 74.010 & $\begin{array}{c}29.993 .459 \\
€\end{array}$ & 270.850 & 198.373 & $\begin{array}{c}42.570 .553 \\
€\end{array}$ \\
\hline $2010(*)$ & 25.540 & $2.856 .908 €$ & 45.740 & 13.868 & $6.190 .940 €$ & 71.280 & 39.408 & $9.047 .848 €$ \\
\hline $\begin{array}{c}\text { TOTAL } \\
2004-2010\end{array}$ & 149.903 & $15.434 .002 €$ & 192.227 & 87.878 & $\begin{array}{c}36.184 .399 \\
€\end{array}$ & 342.130 & 237.781 & $\begin{array}{c}51.618 .401 \\
€\end{array}$ \\
\hline
\end{tabular}

$(*)$ : Datos agregados estimados en proporción a las cifras registradas hasta el 31 de marzo.

Gráfico 3.3:Trabajadores bonificados

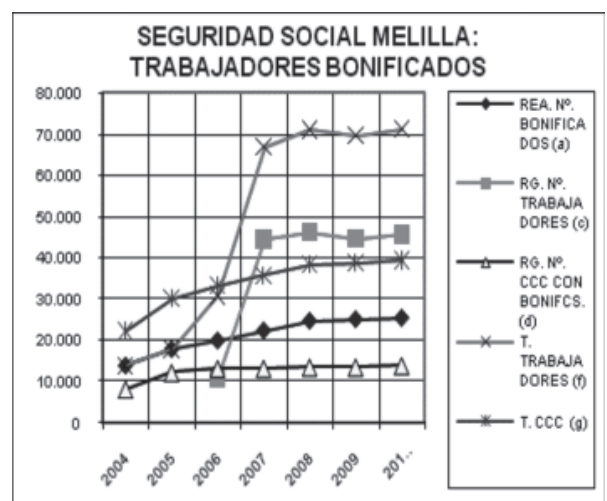

Gráfico 3.4: Importe de bonificaciones

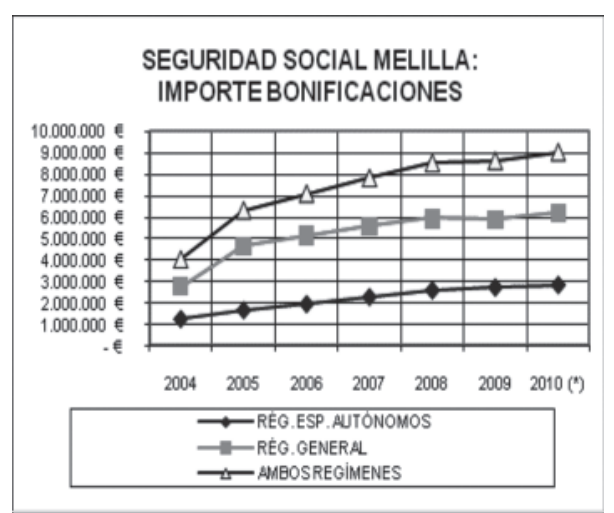

Fuente: Elaboración propia a partir de datos de la Tesorería General de la Seguridad Social (2010) 
Como resulta inmediato, se constata una evolución ascendente tanto en términos de las personas cuyas cuotas han sido bonificadas, como de importes. La diferencia en cuanto a las pendientes de las curvas respectivas (es decir, en cuanto a las tasas de crecimiento) no es significativa, ya que en el caso de los importes, al tratarse de euros corrientes están incorporados fenómenos externos (fundamentalmente el IPC y/o el deflactor del PIB).

Más allá de la citada diferencia de pendientes entre las dos curvas, en ambos casos coinciden las siguientes etapas: hasta finales de 2005 se experimenta una tasa de crecimiento positiva y creciente; a partir de ahí y hasta finales de 2008, si bien la tasa de crecimiento sigue siendo positiva se va desacelerando; desde entonces la curva tiende a hacerse plana (asintótica), lo que significaría que el modelo está agotando actualmente su capacidad de crecimiento.

Tal situación en 2009 y 2010 coincide con lo experimentado en el caso de la evolución de las afiliaciones a la Seguridad Social, ya comentado. En definitiva, volviendo a las bonificaciones en sentido estricto, el modelo parece haber alcanzado su techo de repercusión en trabajadores concernidos y, simultáneamente, en los correspondientes importes.

\subsection{Contrataciones}

En la tabla (3.3) y el gráfico (3.5) posteriores se aportan datos sobre las contrataciones en Melilla registradas en el SPEE $;{ }^{14}$ también en este caso se trata de datos agregados al no disponerse de su desglose por los sectores concernidos.

De entrada, creemos debe prescindirse de los datos correspondientes a abril de 2010, debido a que muy probablemente adolecen de estacionalidad con respecto a los demás períodos considerados: el mes de diciembre en todos los casos. Observando ya el resto de los datos, se constata que tras un primer año de crecimiento positivo y elevado, en el siguiente la tasa decrece, si bien sigue siendo positiva; desde diciembre 2008 la tasa es ya negativa, con una pendiente decreciente cada vez más pronunciada. A pesar de dicha evolución, la tasa de crecimiento acumulado para el período resulta positiva (889 contratos en diciembre 2009 frente a 771 en diciembre 2004).

\footnotetext{
${ }^{14}$ Servicio Público de Empleo Estatal (antes INEM)
} 
Tabla 3.3: Contrataciones registradas

\begin{tabular}{|c|c|c|c|}
\hline \multicolumn{4}{|c|}{$\begin{array}{l}\text { CONTRATACIONES REGISTRADAS (SPEE) } \\
\text { MELILLA }\end{array}$} \\
\hline \multicolumn{4}{|c|}{ NUMERO ABSOLUTO DE PERSONAS } \\
\hline \multirow{3}{*}{ MES-AÑ } & \multicolumn{3}{|c|}{ TOTAL } \\
\hline & $\begin{array}{l}\text { CIFRAS } \\
\text { MES }\end{array}$ & \multicolumn{2}{|c|}{$\begin{array}{l}\text { VARIACIÓN SOBRE } \\
\text { PERÍODO ANTERIOR (*) }\end{array}$} \\
\hline & \multicolumn{2}{|c|}{ Personas } & Porcentajes \\
\hline dic-04 & 771 & & \\
\hline dic-05 & 1.021 & 250 & $32,43 \%$ \\
\hline dic-06 & 1.179 & 158 & $15,48 \%$ \\
\hline dic-07 & 1.163 & -16 & $-1,36 \%$ \\
\hline dic-08 & 1.088 & -75 & $-6,45 \%$ \\
\hline dic-09 & 889 & -199 & $-18,29 \%$ \\
\hline abr- 10 & 1.238 & 349 & $39,26 \%$ \\
\hline
\end{tabular}

\section{Gráfico 3.5: Contrataciones registradas}

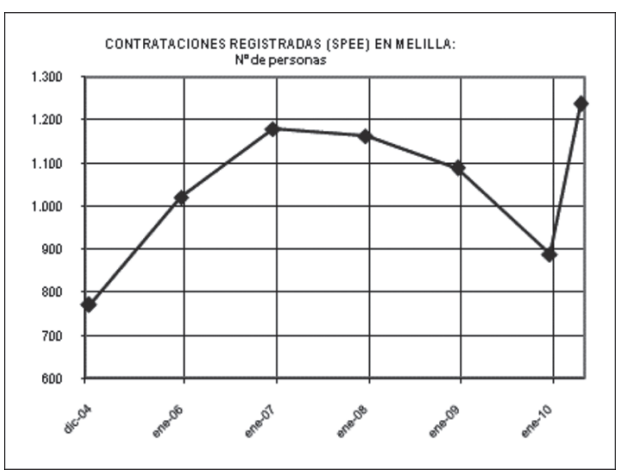

(*) Interanual, excepto en el caso de abril 2010 (sobre diciembre 09)

Fuente: Elaboración propia a partir de datos del INEM y del SPEE (2010)

En un escenario ceteris páribus de todas las variables independientes que, incluida la constituida por la bonificación de cuotas, influyen en la contratación, se comprueba también en este ámbito la validez, en general, de lo ya dicho para los dos casos anteriores.

\subsection{Tasas de actividad}

En la tabla (3.4) y el gráfico (3.6)'ulteriores se aportan datos sobre la evolución de la tasa de actividad registrada en Melilla, y en España en su conjunto, desde el primer trimestre de 2005 hasta el primero de 2010, ambos inclusive.

Como puede comprobarse, la tasa de actividad en Melilla ha sido siempre inferior a la de España, en 6'1 puntos porcentuales diferenciales de media (para los 21 trimestres considerados). La evolución en el período no ha experimentado oscilaciones destacables, con la excepción de algunos picos aislados que van desde un mínimo de 3'34 puntos porcentuales de diferencia (2007TIV), hasta un máximo de 11'11 (2008TIV). Considerando el período por tramos, es de destacar en primer lugar el que finaliza en el tercer trimestre de 2008, dentro del cual se detecta una tendencia de convergencia de la tasa de actividad de Melilla respecto de la nacional; seguidamente se da paso a una evolución ligeramente divergente, que persiste en la actualidad. 
Tabla 3.4:Tasas de actividad

\section{TASAS DE ACTIVIDAD}

\begin{tabular}{|c|c|c|c|}
\hline \multicolumn{4}{|c|}{ TASAS DE ACTIVIDAD } \\
\hline \multirow{2}{*}{$\begin{array}{c}\text { Encuesta de Población } \\
\text { Activa (EPA) }\end{array}$} & $\begin{array}{l}\text { MELILLA } \\
\text { (a) }\end{array}$ & $\begin{array}{l}\text { TOTAL NACIONAL } \\
\text { (b) }\end{array}$ & $\begin{array}{c}\text { TOTAL NACIONAL - } \\
\text { MELILLA } \\
\text { (c) }=(\mathrm{b})-(\mathrm{a})\end{array}$ \\
\hline & Porcentaje & Porcentaje & \begin{tabular}{|l|}
$\begin{array}{l}\text { Puntos porcentuales de } \\
\text { diferencia }\end{array}$ \\
\end{tabular} \\
\hline $2010 \mathrm{TI}$ & 53,03 & 59,83 & 6,80 \\
\hline 2009TIV & 51,72 & 59,76 & 8,04 \\
\hline 2009TIII & 52,10 & 59,81 & 7,71 \\
\hline 2009TII & 54,82 & 60,06 & 5,24 \\
\hline $2009 \mathrm{TI}$ & 52,83 & 60,15 & 7,32 \\
\hline 2008TIV & 49,02 & 60,13 & 11,11 \\
\hline 2008TIII & 55,31 & 59,95 & 4,64 \\
\hline 2008TII & 54,39 & 59,76 & 5,37 \\
\hline $2008 \mathrm{TI}$ & 54,65 & 59,35 & 4,70 \\
\hline 2007TIV & 55,78 & 59,12 & 3,34 \\
\hline 2007TIII & 55,59 & 59,10 & 3,51 \\
\hline 2007TII & 53,96 & 58,86 & 4,90 \\
\hline $2007 \mathrm{TI}$ & 50,94 & 58,58 & 7,64 \\
\hline 2006TIV & 51,41 & 58,58 & 7,17 \\
\hline 2006TIII & 53,53 & 58,44 & 4,91 \\
\hline 2006TII & 51,63 & 58,30 & 6,67 \\
\hline $2006 \mathrm{TI}$ & 53,43 & 57,98 & 4,55 \\
\hline 2005 TIV & 51,38 & 57,72 & 6,34 \\
\hline 2005TIII & 48,83 & 57,43 & 8,60 \\
\hline 2005TII & 51,84 & 57,35 & 5,51 \\
\hline $2005 \mathrm{TI}$ & 52,67 & 56,90 & 4,23 \\
\hline
\end{tabular}

\section{Gráfico 3.6:Tasas de actividad}

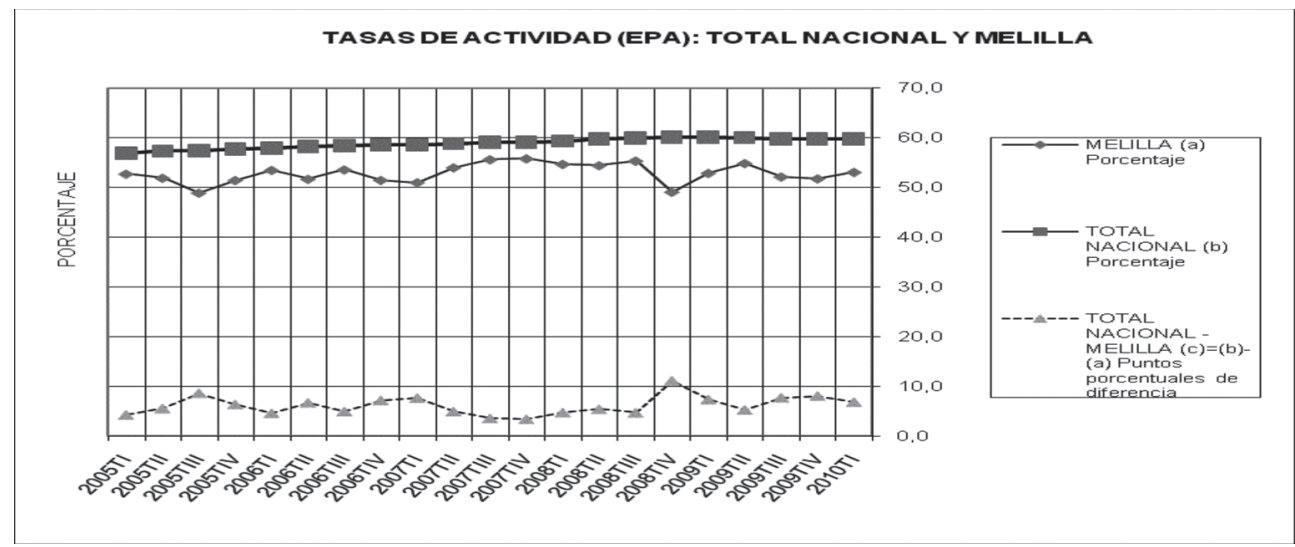

Nota.- Tasa de actividad: Porcentaje de activos respecto de la población total.

Fuente: Elaboración propia a partir de datos del INE (2010) 
Si introducimos en el análisis la consideración de la evolución de la población total, por ser una de las dos variables que conforman la tasa de actividad (la otra es obviamente la población activa), comprobamos que la tasa acumulada de crecimiento de la población entre 2004 y 2009 es del 7’6\% en el caso de España y del 5’1\% en el caso de Melilla. El efecto inmediato es que si la población de Melilla hubiera crecido a la tasa más alta del conjunto de España, las tasas de actividad de Melilla habrían sido inferiores a las efectivamente registradas, con lo que, por otra parte, habrían tenido una mayor divergencia respecto de las tasas de España.

A pesar de no poderse documentar una correlación positiva entre el aumento de bonificaciones en Melilla y la evolución de la tasa de actividad, al haber permanecido esta casi invariable a lo largo del período considerado, debe tenerse en cuenta un hecho de interés para un análisis alternativo. Se trata de que, al haber experimentado la población total de Melilla un crecimiento acumulado entre 2004 y 2009 del 5\%1\%, se tiene que haber producido una absorción de ese incremento de población total por parte de la población activa (al haber permanecido esta casi invariable en términos de tasa); dicho de otra manera, si pasamos de términos de tasa de población activa (casi invariable) a términos absolutos, las personas activas han debido incrementarse en dicho período en, aproximadamente, el 5’1\% antes citado, es decir, en unas 3.418 personas. Siguiendo este razonamiento, se ha procedido a calcular la correlación entre la evolución del importe de las bonificaciones entre 2004 y 2009 y la evolución de las personas activas en una senda coincidente con la evolución experimentada por la evolución de la población total de Melilla; pues bien, el resultado es un Coeficiente de Correlación de 0'767968 (con un Coeficiente R2 de 0'589775), no demasiado elevado pero tampoco desdeñable.

\subsection{Tasas de empleo}

En la tabla (3.5) y el gráfico (3.7) siguientes se aportan datos sobre la evolución de la tasa de empleo registrada en Melilla, y en España en su conjunto, desde el primer trimestre de 2005 hasta el primero de 2010, ambos inclusive. 
Tabla 3.5: Tasas de empleo

\begin{tabular}{|c|c|c|c|}
\hline \multirow{2}{*}{$\begin{array}{c}\text { Encuesta de Población } \\
\text { Activa (EPA) }\end{array}$} & $\begin{array}{c}\text { MELILLA } \\
\text { (a) }\end{array}$ & $\begin{array}{l}\text { TOTAL NACIONAL } \\
\text { (b) }\end{array}$ & $\begin{array}{l}\text { TOTAL NACIONAL - } \\
\text { MELILLA } \\
\text { (c) }=(\mathrm{b})-(\mathrm{a})\end{array}$ \\
\hline & Porcentaje & Porcentaje & $\begin{array}{l}\text { Puntos porcentuales de } \\
\text { diferencia }\end{array}$ \\
\hline 2010TI & 41,88 & 47,84 & 5,96 \\
\hline 2009TIV & 40,51 & 48,50 & 7,99 \\
\hline 2009TIII & 39,92 & 49,09 & 9,17 \\
\hline 2009TII & 40,45 & 49,29 & 8,84 \\
\hline 2009TI & 39,42 & 49,70 & 10,28 \\
\hline 2008TIV & 40,75 & 51,77 & 11,02 \\
\hline 2008TIII & 42,16 & 53,16 & 11,00 \\
\hline 2008TII & 44,04 & 53,52 & 9,48 \\
\hline $2008 \mathrm{TI}$ & 42,22 & 53,63 & 11,41 \\
\hline 2007TIV & 46,01 & 54,03 & 8,02 \\
\hline 2007TIII & 45,42 & 54,36 & 8,94 \\
\hline 2007TII & 42,62 & 54,18 & 11,56 \\
\hline $2007 \mathrm{TI}$ & 42,90 & 53,62 & 10,72 \\
\hline 2006TIV & 46,41 & 53,72 & 7,31 \\
\hline 2006TIII & 46,91 & 53,68 & 6,77 \\
\hline $2006 \mathrm{TII}$ & 44,11 & 53,32 & 9,21 \\
\hline $2006 \mathrm{TI}$ & 44,44 & 52,72 & 8,28 \\
\hline 2005TIV & 45,30 & 52,70 & 7,40 \\
\hline 2005TIII & 43,25 & 52,59 & 9,34 \\
\hline $2005 \mathrm{TII}$ & 44,05 & 52,00 & 7,95 \\
\hline 2005 TI & 43,50 & 51,10 & 7,60 \\
\hline
\end{tabular}

\section{Gráfico 3.7:Tasas de empleo}

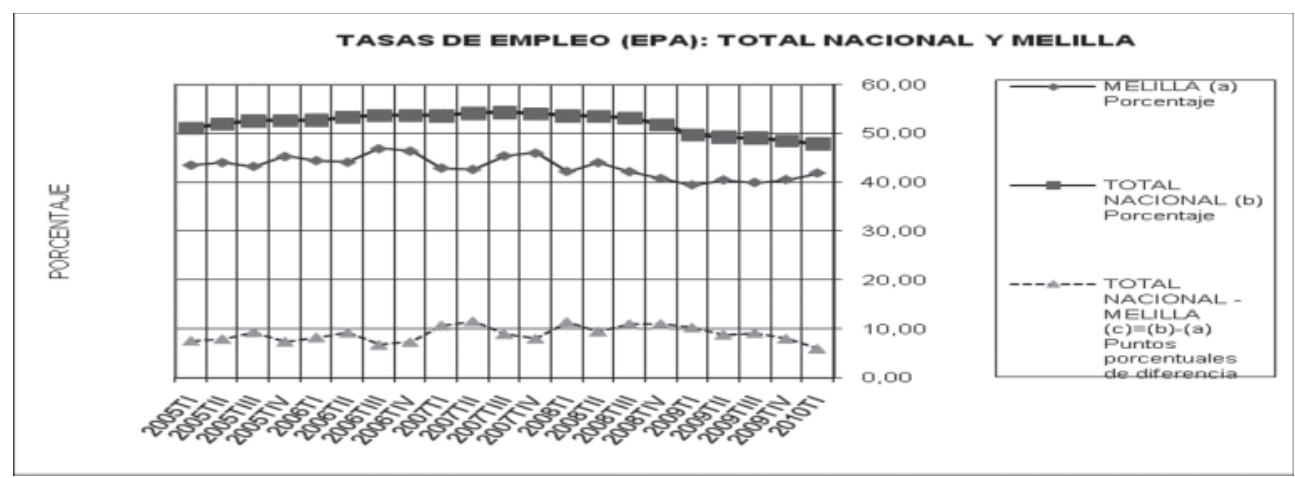

Nota.- Tasa de empleo: Porcentaje de ocupados respecto de la población total.

Fuente: Elaboración propia a partir de datos del INE (2010) 
Si nos centramos en la evolución de la tasa de empleo en Melilla, prescindiendo de la nacional, se comprueba que para el período comprendido entre 2005 y 2009 (único para el que existen datos completos que permitan el correspondiente análisis), ha existido una correlación negativa, si bien no significativa, entre el importe de las bonificaciones y la tasa de empleo; en efecto, según los cálculos efectuados, el correspondiente Coeficiente de Correlación se sitúa en -0'781544 (que a su vez presenta un Coeficiente R2 de 0’610811). ${ }^{15}$

Pasando ahora a términos comparativos entre Melilla y el total de España se observa lo siguiente: a) La tasa de empleo en Melilla ha sido siempre inferior a la de España, en 9'0 puntos porcentuales diferenciales de media (para los 21 trimestres considerados); b) La evolución en el período no ha experimentado oscilaciones destacables, con la excepción de algunos picos aislados que van desde un máximo de 11'56 puntos porcentuales de diferencia (2007TII), hasta un mínimo de 5’96 (2010TI); c) Considerando el período por tramos, es de destacar en primer lugar el que finaliza en el primer trimestre de 2009, dentro del cual se detecta una tendencia de ligera divergencia de la tasa de empleo de Melilla respecto de la nacional; seguidamente se da paso a una evolución ligeramente convergente, que persiste en la actualidad.

Si al igual que se ha hecho en el epígrafe anterior se prescinde del análisis en términos de tasa, por su escasa utilidad al poder enmascarar aumentos de personas ocupadas simultáneos al de población total, y se efectúa un análisis alternativo a partir de valores absolutos, es decir, de número de personas ocupadas, la correlación que resulta entre las bonificaciones vigentes y las personas ocupadas es positiva pero sin entidad suficiente; en efecto, el Coeficiente de Correlación se queda en 0,362688 (con un Coeficiente R2 de 0'135428).

\subsection{Paro}

En las tablas (3.6 y 3.7) y gráficos (3.8 y 3.9) siguientes se aportan datos sobre la evolución del paro en Melilla, así como en España en su conjunto, desde el primer trimestre de 2005 hasta el primero de 2010, ambos inclusive.

\footnotetext{
${ }^{15}$ Los importes utilizados en el cálculo son los que se recogen en el apartado 3.2 (Bonificaciones) y las tasas de empleo incluidas en este.
} 
Tabla 3.6: Paro registrado

\begin{tabular}{|c|c|c|c|}
\hline \multicolumn{3}{|c|}{ PARO REGISTRADO EN } & MELILLA (SPEE) \\
\hline \multicolumn{3}{|c|}{ NUMERO ABSOLUTO DE PERSONAS } \\
\hline \multirow{2}{*}{$\begin{array}{c}\text { MES-AÑ } \\
\text { O }\end{array}$} & $\begin{array}{c}\text { CIFRAS } \\
\text { MES }\end{array}$ & $\begin{array}{c}\text { VARIACIÓN SOBRE } \\
\text { PERÍODO ANTERIOR (*) }\end{array}$ \\
\cline { 2 - 4 } & \multicolumn{2}{|c|}{ Personas } & $\%$ \\
\hline dic-04 & 4.568 & & $53,63 \%$ \\
\hline dic-05 & 7.018 & 2.450 & $-13,42 \%$ \\
\hline dic-06 & 6.076 & -942 & $5,51 \%$ \\
\hline dic-07 & 6.411 & 335 & $20,25 \%$ \\
\hline dic-08 & 7.709 & 1.298 & $8,33 \%$ \\
\hline dic-09 & 8.351 & 642 & $12,78 \%$ \\
\hline abr-10 & 9.418 & 1.067 & \\
\hline
\end{tabular}

\section{Gráfico 3.8: Paro registrado}

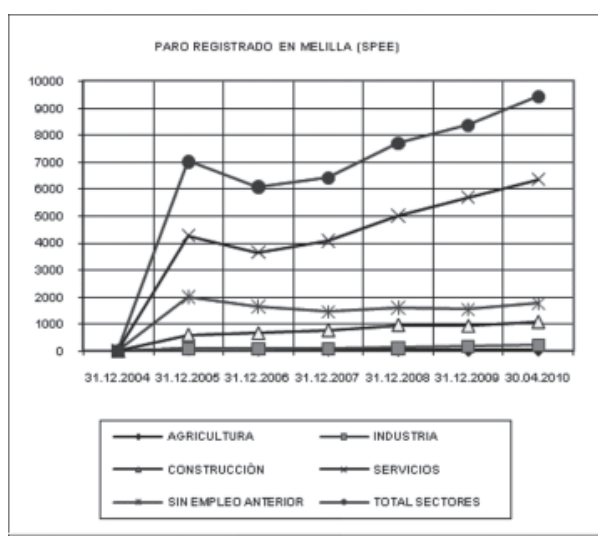

(*) Interanual, excepto en el caso de abril 2010 (sobre diciembre 09)

Fuente: Elaboración propia a partir de datos del INEM y del SPEE (2010)

Tabla 3.7:Tasas de paro

\begin{tabular}{|c|c|c|c|}
\hline \multicolumn{4}{|c|}{ TASA DE PARO } \\
\hline \multirow{2}{*}{$\begin{array}{c}\text { Encuesta de Población } \\
\text { Activa (EPA) }\end{array}$} & $\begin{array}{l}\text { MELILLA } \\
\text { (a) }\end{array}$ & $\begin{array}{l}\text { TOTAL NACIONAL } \\
\text { (b) }\end{array}$ & $\begin{array}{c}\text { MELILLA - } \\
\text { TOTAL NACIONAL } \\
\text { (c) }=(\text { b) }- \text { (a) }\end{array}$ \\
\hline & Porcentaje & Porcentaje & $\begin{array}{l}\text { Puntos porcentuales de } \\
\text { diferencia }\end{array}$ \\
\hline 2010TI & 21,02 & 20,05 & 0,97 \\
\hline 2009TIV & 21,68 & 18,83 & 2,85 \\
\hline 2009TIII & 23,39 & 17,93 & 5,46 \\
\hline 2009TII & 26,22 & 17,92 & 8,30 \\
\hline $2009 \mathrm{TI}$ & 25,39 & 17,36 & 8,03 \\
\hline 2008 TIV & 16,87 & 13,91 & 2,96 \\
\hline 2008TIII & 23,77 & 11,33 & 12,44 \\
\hline 2008TII & 19,04 & 10,44 & 8,60 \\
\hline $2008 \mathrm{TI}$ & 22,74 & 9,63 & 13,11 \\
\hline 2007 TIV & 17,52 & 8,60 & 8,92 \\
\hline 2007TIII & 18,29 & 8,03 & 10,26 \\
\hline $2007 \mathrm{TII}$ & 21,02 & 7,95 & 13,07 \\
\hline $2007 \mathrm{TI}$ & 15,79 & 8,47 & 7,32 \\
\hline 2006TIV & 9,73 & 8,30 & 1,43 \\
\hline 2006TIII & 12,37 & 8,15 & 4,22 \\
\hline 2006TII & 14,57 & 8,53 & 6,04 \\
\hline $2006 \mathrm{TI}$ & 16,82 & 9,07 & 7,75 \\
\hline 2005 TIV & 11,83 & 8,70 & 3,13 \\
\hline 2005TIII & 11,43 & 8,42 & 3,01 \\
\hline 2005TII & 15,03 & 9,33 & 5,70 \\
\hline 2005TI & 17,41 & 10,19 & 7,22 \\
\hline
\end{tabular}




\section{Gráfico 3.9: Tasas de paro}

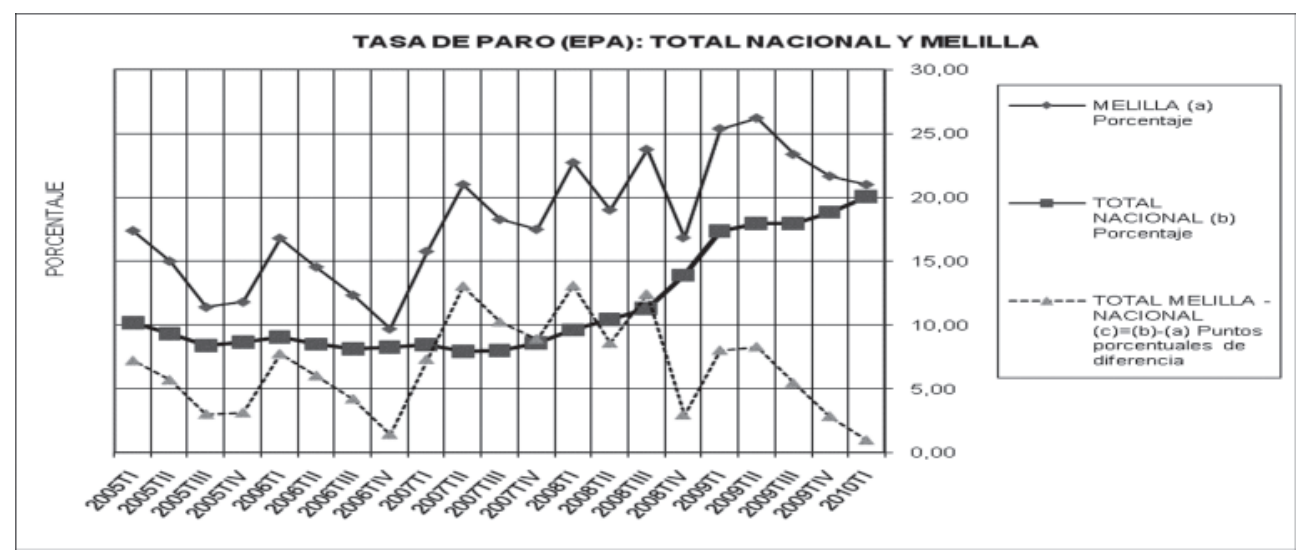

Nota.- Tasa de paro: Porcentaje de parados respecto de la población activa.

Fuente: Elaboración propia a partir de datos del INE (2010)

En términos comparativos con el total de España, los 7'22 puntos porcentuales con que la tasa de paro de Melilla, en términos de la EPA, ${ }^{16}$ superaba a España en 2005, tras una serie de altibajos se han reducido en 2010 (primer trimestre) a 0'97. La convergencia se está produciendo, no obstante, no en el entorno de partida de España en 2005: una tasa de paro del 10'19 (el 17’41\% en Melilla), sino en el entorno alcanzado por España (tras un período de crecimiento ininterrumpido de dicha tasa comenzado en el segundo semestre de 2007) en el primer trimestre de 2010 (20’05\% España y 21'02\% Melilla).

Si prescindimos de comparaciones, puede observarse que la tasa de paro en Melilla ha mantenido desde 2005 hasta hoy, en términos generales y con independencia de los altibajos intermedios registrados, una evolución creciente. Se ha pasado, como acaba de señalarse, de una tasa del 17'41\% en 2005T1 a otra del 21'02\% en 2010T1.

Por otra parte y como puede comprobarse asimismo, la contribución de los diferentes sectores económicos al paro registrado en Melilla es, en valores absolutos y por orden de menos a más, la siguiente: $1^{\circ}$ ) Agricultura; $2^{\circ}$ ) Industria; $3^{\circ}$ ) Construcción; $4^{\circ}$ ) Grupo de personas sin empleo anterior; $5^{\circ}$ ) Servicios. Es precisamente este último

\footnotetext{
${ }^{16}$ Encuesta de Población Activa, sobre la que el INE advierte de que en el caso de Melilla y Ceuta debe tomarse con precaución, al poder estar afectada por errores de muestreo.
} 
sector el que destaca sobre los demás en cuanto a crecimiento de personas paradas a lo largo del período considerado.

Pese a la convergencia señalada de la tasa de paro de Melilla respecto de la de España, se da la paradoja de que, al haberse alcanzado tras un período de crecimiento neto de ambas tasas, no es posible documentar una correlación negativa entre el aumento de bonificaciones en Melilla y la evolución de la tasa de paro. Empero, si recurrimos a la evolución de las diferencias porcentuales entre la tasa de paro Melilla-España entre 2005 y 2010 (utilizando los datos del cuarto trimestre entre 2005 y 2009 y del primero en 2010), la reducción de dicho diferencial (supuesta variable dependiente), simultáneamente a la coexistencia de bonificaciones de cuotas a la Seguridad Social en Melilla (supuesta variable independiente), comporta a simple vista una correlación negativa. Pues bien, efectuados los cálculos correspondientes, el resultado es, en efecto, un Coeficiente de Correlación negativo: - 0,121669 (con un Coeficiente R2 0,014803); sin embargo, la proximidad al cero de ambos coeficientes viene a indicar la práctica ausencia de correlación significativa entre las variables analizadas.

\section{EVALUACIÓN PROPIA DE OTRAS VARIABLES}

El capítulo anterior ha sido dedicado a la evaluación, en el marco prevenido por la normativa vigente y, en particular, por la ORDENTIN/530/23010, del grado de eficacia de las bonificaciones con relación a los objetivos sociales que se pretenden alcanzar. Sin embargo, dichos objetivos no son los únicos a considerar, habida cuenta que existen otros valores sociales que pueden estar correlacionados, en principio, con las bonificaciones adoptadas. En consecuencia, el presente apartado va a dedicarse al examen de determinados objetivos sociales adicionales a los contemplados hasta ahora.

\subsection{Convenios colectivos}

En ausencia de datos sobre convenios colectivos en Melilla referidos a los distintos sectores beneficiados por las bonificaciones, van a utilizarse los datos agregados que, correspondientes a Melilla y Ceuta conjuntamente, por una parte, y a España en general, por otra, se ofrecen en la tabla (3.8) y el gráfico (3.10) que se insertan seguidamente. 
Tabla 3.8: Convenios colectivos

\section{Convenios colectivos (I)}

$\%$ de trabajadores afectados sobre afiliados a la SS (11)

\begin{tabular}{|l|r|r|r|r|r|}
\hline & \multicolumn{5}{|c|}{ Porcentaje } \\
\hline & 2004 & 2005 & 2006 & 2007 & 2008 \\
\hline Total España & 74,08 & 75,07 & 74,29 & 74,92 & 70,02 \\
\hline Ceuta y Melilla & 38,64 & 40,09 & 33,25 & 24,57 & 20,95 \\
\hline Ceuta & $\ldots$ & $\ldots$ & $\ldots$ & $\ldots$ & $\ldots$ \\
\hline Melilla & $\ldots$ & $\ldots$ & $\ldots$ & $\ldots$ & $\ldots$ \\
\hline
\end{tabular}

\section{Convenios colectivos (II)}

$\%$ de aumento salarial revisado (12)

\begin{tabular}{|l|r|r|r|r|r|}
\hline & \multicolumn{5}{|c|}{ Porcentaje } \\
\hline & 2004 & 2005 & 2006 & 2007 & 2008 \\
\hline Total España & 3,60 & 4,04 & 3,59 & 4,21 & 3,55 \\
\hline Ceuta y Melilla & 3,54 & 3,87 & 3,60 & 3,66 & 4,71 \\
\hline Ceuta & $\ldots$ & $\ldots$ & $\ldots$ & $\ldots$ & $\ldots$ \\
\hline Melilla & $\ldots$ & $\ldots$ & $\ldots$ & $\ldots$ & $\ldots$ \\
\hline
\end{tabular}

\section{Gráfico 3.10: Convenios colectivos}
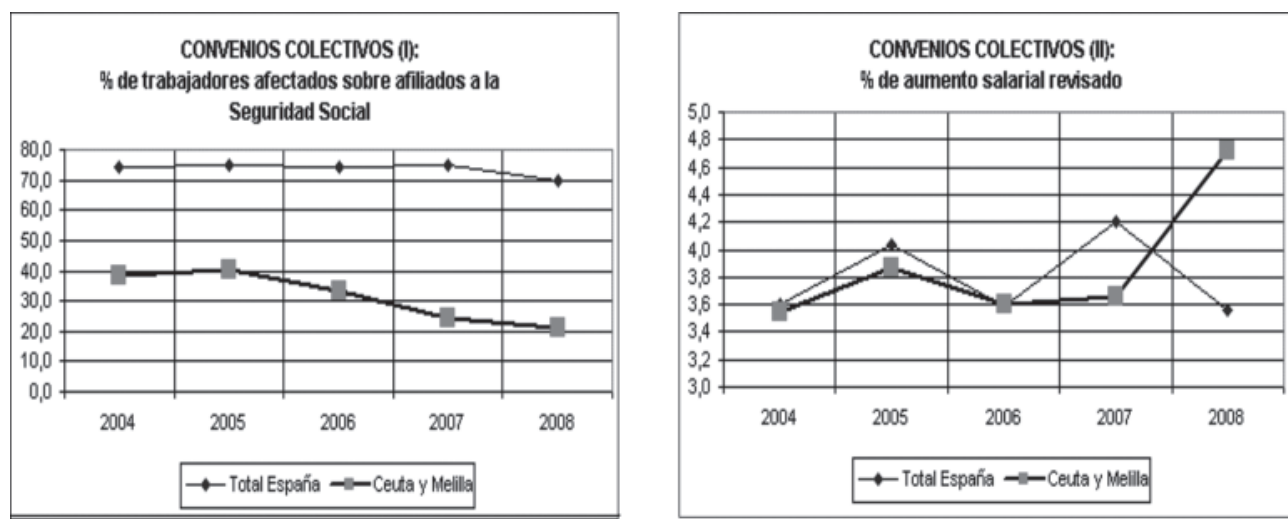

(11) Incluye los afiliados al régimen general y a los regímenes especiales de la minería del carbón, el agrario por cuenta ajena y el del mar por cuenta ajena.

(11) y (12) Los años son los del inicio de los efectos económicos.

Fuente: Elaboración propia a partir de datos del INE (2010)

Como puede verificarse, la adopción de las bonificaciones en las cuotas no se ha traducido en un aumento de la relación entre los trabajadores afectados por los diferentes convenios colectivos y el total de trabajadores afiliados a la Seguridad Social. Si, por otra parte, comparamos la evolución registrada en Melilla y Ceuta en este aspecto, con la evolución del total nacional, comprobamos que el diferencial en contra de Melilla y Ceuta se ha ido agrandando, pasando de 35,4 puntos porcentuales en 2004 a 49, 1 en 2008. En definitiva, la adopción de las bonificaciones no ha servido en Melilla y Ceuta para la extensión de convenios colectivos entre los trabajadores afiliados a la Seguridad Social. 
Por el contrario, en términos de porcentaje de aumento salarial revisado sí se ha registrado un aumento en Melilla y Ceuta a partir de 2006, débil en 2007 y pronunciado en 2008. Dicha situación positiva contrasta con la experimentada en sentido contrario en el ámbito nacional; en efecto, Melilla y Ceuta han pasado de registrar en 2004 un diferencial negativo de 0'06 puntos porcentuales con respecto a España en su conjunto, a un diferencial positivo de 1'16 en 2008. La correlación entre las bonificaciones y los aumentos salariales calculada para el período del que se dispone de suficientes datos: 2004-2008, si bien ha sido de signo positivo es escasamente significativa; en efecto, los cálculos efectuados determinan un Coeficiente de Correlación de tan sólo 0'618319 (que a su vez cuenta con un Coeficiente R2 de 0,382318). ${ }^{17}$

En consecuencia, si bien es cierto que las bonificaciones no se han traducido en una mayor cobertura de los trabajadores mediante convenios colectivos, no lo es menos que el porcentaje de aumento salarial revisado sí ha crecido.

\subsection{Remuneraciones por asalariado}

A continuación van a insertarse las tablas (3.9) y los gráficos (3.11) elaborados con la remuneración de los asalariados por sectores y subsectores (ramas) de actividad, en términos del incremento interanual acumulado entre 2004 y 2007. Los datos se refieren tanto a Melilla en particular como al total de España.

Empezando por un ámbito desagregado: los subsectores englobados en el sector servicios, destacan los servicios de hostelería, en tanto las remuneraciones por asalariado en Melilla han experimentado un retroceso acumulado, en el período 2004-2007, del $11^{\prime} 4 \%$ (el 9'4\% en el caso del total de España). Para el total de los sectores, el incremento anual acumulado por asalariado para el período 2004-2007 ha sido en Melilla del 16’5\%, frente al 10,5\% en el caso del total de España.

${ }^{17}$ Los importes utilizados en el cálculo son los que se recogen en el apartado 3.2 (Bonificaciones) y los porcentajes de aumento salarial incluidos en este. 


\begin{tabular}{|c|c|c|}
\hline \multicolumn{3}{|c|}{$\begin{array}{c}\text { REMUNERACIÓN DE LOS } \\
\text { ASALARIADOS POR SECTORES DE } \\
\text { ACTIVIDAD: } \\
\text { INCREMENTO ACUMULADO } \\
(\mathbf{2 0 0 7 / 2 0 0 4 ) ~ P O R ~ A S A L A R I A D O ~}\end{array}$} \\
\hline & MELILLA & ESPAÑA \\
\hline $\begin{array}{l}\text { A gricultura, ganadería } \\
\text { y pesca }\end{array}$ & $50,0 \%$ & $14,9 \%$ \\
\hline Energía & $6,1 \%$ & $12,4 \%$ \\
\hline Industria & $-7,1 \%$ & $14,0 \%$ \\
\hline Construcción & $16,3 \%$ & $13,3 \%$ \\
\hline Servicios & $16,9 \%$ & $9,0 \%$ \\
\hline
\end{tabular}

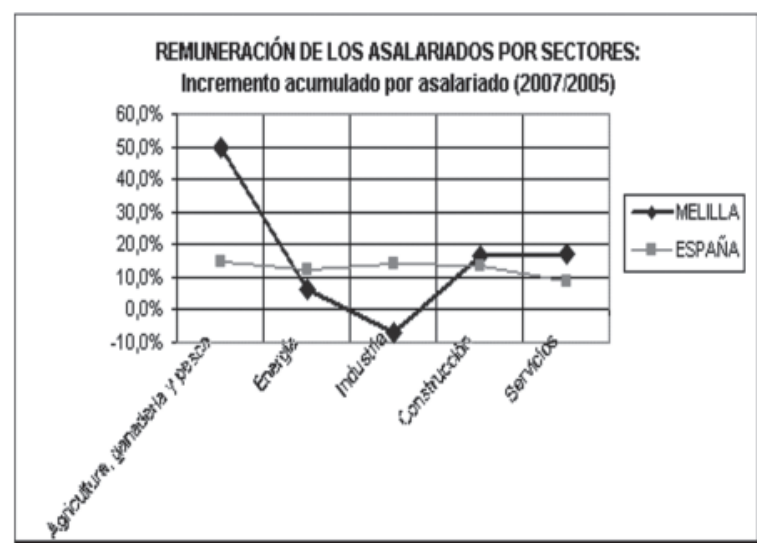

\section{REMUNERACIÓN DE LOS \\ ASALARIADOS POR SUBSECTORES DE SERVICIOS:}

INCREMENTO ACUMULADO (2007/2004) POR ASALARIADO

\begin{tabular}{|c|c|c|}
\hline & MELILLA & ESPAÑA \\
\hline $\begin{array}{l}\text { GG Comercio y } \\
\text { reparación }\end{array}$ & $15,5 \%$ & $10,1 \%$ \\
\hline HH Hostelería & $-11,4 \%$ & $-9,4 \%$ \\
\hline $\begin{array}{l}\text { II Transporte y } \\
\text { comunicaciones }\end{array}$ & $28,2 \%$ & $8,3 \%$ \\
\hline $\begin{array}{l}\text { JJ Intermediación } \\
\text { financiera }\end{array}$ & $5,8 \%$ & $14,5 \%$ \\
\hline $\begin{array}{l}\text { KK Inmobiliarias y } \\
\text { servicios } \\
\text { empresariales }\end{array}$ & $12,2 \%$ & $6,1 \%$ \\
\hline $\begin{array}{l}\text { LL Administración } \\
\text { pública }\end{array}$ & $13,9 \%$ & $21,1 \%$ \\
\hline MM Educación & $28,2 \%$ & $9,8 \%$ \\
\hline $\begin{array}{l}\text { NN Actividades } \\
\text { sanitarias y } \\
\text { veterinarias; servicios } \\
\text { sociales }\end{array}$ & \multirow[t]{2}{*}{$35,2 \%$} & \multirow[t]{2}{*}{$11,0 \%$} \\
\hline $\begin{array}{l}\text { OO Otros servicios y } \\
\text { actividades sociales; } \\
\text { servicios personales }\end{array}$ & & \\
\hline $\begin{array}{l}\text { PP Hogares que } \\
\text { emplean personal } \\
\text { doméstico }\end{array}$ & $5,1 \%$ & $5,5 \%$ \\
\hline
\end{tabular}

\section{REAUNERACIONOLELOS ASALAARADOS POR SUBSECTORES SERVICIOS:} Incremento gcunulado por assalariado

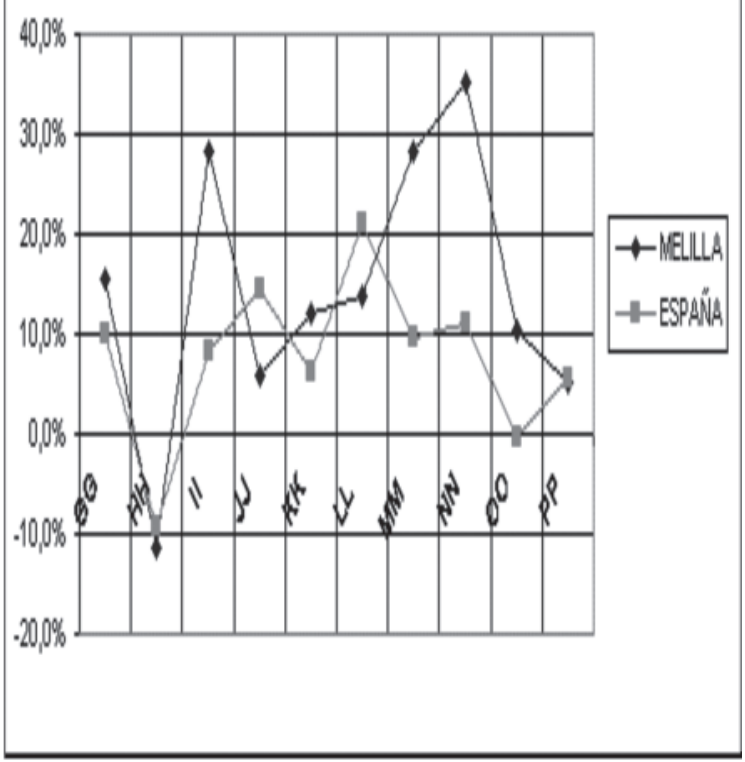



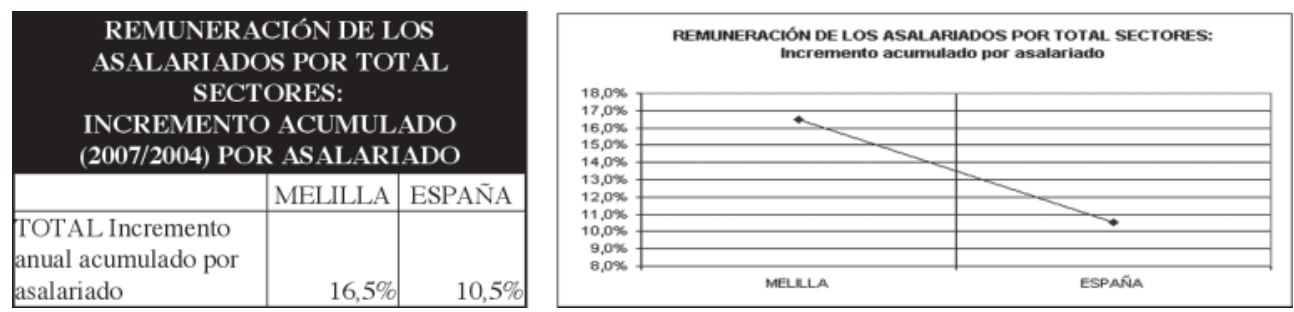

Los datos de 2007 son una estimación provisional; los de 2004 a 2006 son definitivos.

Fuente: Elaboración propia a partir de datos del INE (2010)

Centrándonos ya en el ámbito de los sectores, y tras prescindir del examen del primario debido a que al ser sus magnitudes muy pequeñas los resultados pueden adolecer de escasa representatividad, destaca el hecho de que, en el período 2004-2007, las remuneraciones por asalariado de la industria han descendido en Melilla en un 7'1\%, en tanto que en el total de España han aumentado en un 14'0\%. En el sector de la energía se contrapone un incremento del 6'1\% en Melilla frente al 12'4\% en España. Por su parte, en el sector de los servicios sobresale un incremento del 16'9\% en Melilla frente al 9'0\% en España. Por último, en el sector de la construcción no existen diferencias tan significativas, al registrarse un incremento del 16’3\% en Melilla frente al 13’3\% en España.

\subsection{Contratación indefinida}

Posteriormente se insertan las tablas (3.10) y los gráficos (3.12) elaborados a partir de la información disponible sobre los contratos registrados de duración indefinida, distinguiendo dos variables: por una parte, el porcentaje de los contratos registrados de duración indefinida con respecto al total de contratos; y por otro lado, el porcentaje de asalariados con contrato indefinido respecto del total de asalariados. Los ámbitos seleccionados han sido Melilla y Ceuta (sólo se ha dispuesto de datos conjuntos) y el total de España.

En cuanto al porcentaje de contratos registrados de duración indefinida, se documenta un alza entre 2004 (6’96\%) y 2006 (10’67\%). A partir de entonces se detecta un descenso que lleva el porcentaje hasta el 8'49, por encima, no obstante, del nivel de partida en 2004. En términos comparativos con el total de España, en ambos espacios se registra una evolución similar, si bien Melilla siempre por debajo y, desde 2006, con un diferencial negativo cada vez mayor.

En lo atinente al porcentaje de asalariados con contrato indefinido, en Melilla se ha registrado un crecimiento sostenido entre 2004 (62’69\%) y 2007 (67’13\%). A partir de 
entonces se detecta un descenso paulatino que lleva el porcentaje hasta el 65'55, por encima, no obstante, del nivel de partida en 2004. En términos comparativos con el total de España, en ambos espacios se registra una evolución inversa; así, la práctica convergencia que se había conseguido en 2006, se torna a continuación en divergencia, de manera que en 2008 el diferencial negativo de Melilla se sitúa en 5’19 puntos porcentuales (4'86 en 2004).

\section{Tabla 3.10: Contratos registrados de duración indefinida}

CONTRATOS REGISTRADOS DE DURACIÓN
INDEFINIDA (I)
\begin{tabular}{|l|r|r|r|r|r|}
\hline \% de contratos registrds. de duración indefinida (7) \\
\hline & $\mathbf{2 0 0 4}$ & $\mathbf{2 0 0 5}$ & $\mathbf{2 0 0 6}$ & $\mathbf{2 0 0 7}$ & $\mathbf{2 0 0 8}$ \\
\hline España & 8,68 & 8,99 & 11,75 & 11,92 & 11,46 \\
\hline $\begin{array}{l}\text { Ceuta y } \\
\text { Melilla }\end{array}$ & 6,96 & 8,57 & 10,67 & 9,92 & 8,49 \\
\hline
\end{tabular}

\begin{tabular}{|l|c|c|c|c|c|}
\hline \multicolumn{7}{|c|}{ CONTRATOS REGISTRADOS DE DURACIÓN } \\
INDEFINIDA (II) \\
\hline \multicolumn{6}{|c|}{ \% de asalariados con contrato indefinido } \\
\hline & $\mathbf{2 0 0 4}$ & $\mathbf{2 0 0 5}$ & $\mathbf{2 0 0 6}$ & $\mathbf{2 0 0 7}$ & $\mathbf{2 0 0 8}$ \\
\hline Total España & 67,55 & 66,66 & 65,96 & 68,34 & 70,74 \\
\hline Ceuta y Melilla & 62,69 & 62,72 & 66,58 & 67,13 & 65,55 \\
\hline
\end{tabular}

\section{Gráfico 3.12: Contratos registrados de duración indefinida}
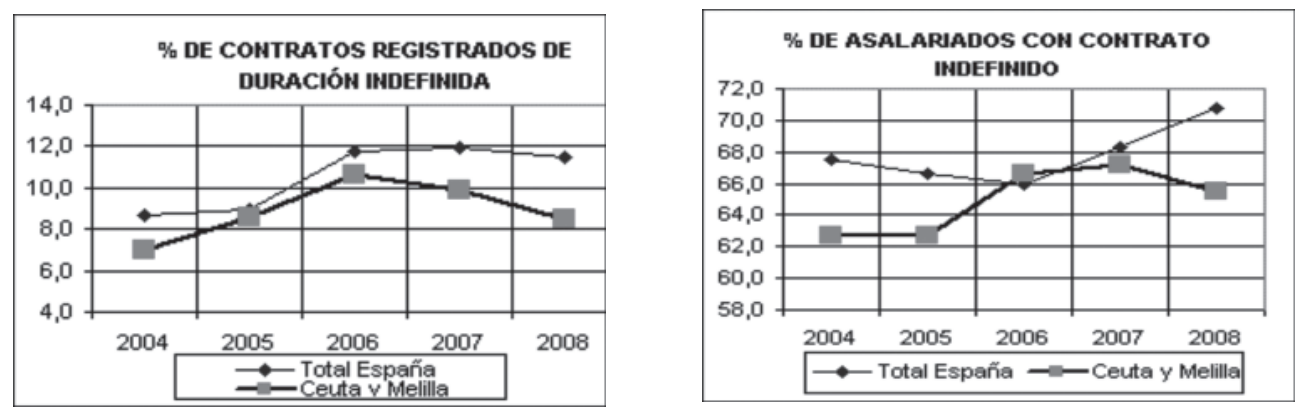

Fuente: Elaboración propia a partir de datos del INE (2010)

Calculada la correlación entre las bonificaciones de cuotas y las variables acabadas de comentar, para el período 2004-2008, los resultados son: a) En el caso del porcentaje de contratos registrados de duración indefinida, el Coeficiente de Correlación es positivo, si bien poco significativo: en concreto 0'646129 (con un Coeficiente R2 de 0,417483); b) En el caso del porcentaje de asalariados con contrato indefinido, el Coeficiente de Correlación es también positivo, más significativo que el anterior pero tampoco demasiado: en concreto 0'757711 (con un Coeficiente R2 de 0,574125). 


\subsection{PIB por habitante y VAB por sectores}

Seguidamente se inserta la tabla (3.11) y el gráfico (3.13), elaborados a partir de la información disponible sobre la evolución del Producto Interior Bruto (PIB) por habitante, para Melilla y el total de España en el período 2004-2008.

Tabla 3.11: PIB

\begin{tabular}{|l|c|c|c|c|c|}
\hline \multicolumn{5}{|c|}{ PRODUCTO INTERIOR BRUTO } \\
\hline \multicolumn{4}{|c|}{ Importes a precios de mercado (1): Euros por habitante } \\
\hline & $\mathbf{2 0 0 4}$ & $\mathbf{2 0 0 5}$ & $\mathbf{2 0 0 6}$ & $\mathbf{2 0 0 7}$ & $\mathbf{2 0 0 8}$ \\
\hline Total España & 19.700 & 20.941 & 22.335 & 23,460 & 23.874 \\
\hline $\begin{array}{l}\text { Incremento/año } \\
\text { España }\end{array}$ & & $6,3 \%$ & $6,7 \%$ & $5,0 \%$ & $1,8 \%$ \\
\hline $\begin{array}{l}\text { Incremento } \\
\text { acumulado } \\
\text { España }\end{array}$ & 17.155 & 18.592 & 20.170 & 21.085 & 21.555 \\
\hline $\begin{array}{l}\text { Total Melilla } \\
\text { Incremento/año } \\
\text { Melilla }\end{array}$ & & $8,4 \%$ & $8,5 \%$ & $4,5 \%$ & $2,2 \%$ \\
\hline $\begin{array}{l}\text { Incremento } \\
\text { acumulado } \\
\text { Melilla }\end{array}$ & $8,4 \%$ & $17,6 \%$ & $22,9 \%$ & $25,6 \%$ \\
\hline
\end{tabular}

Gráfico 3.13: PIB

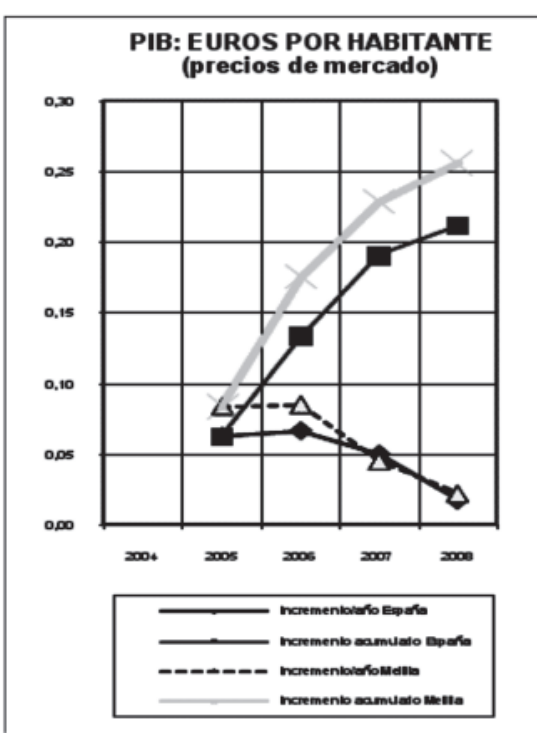

(1) La población utilizada para calcular el PIB por habitante está calculada a 1 de julio. Cifras revisadas en base al Censo de 2001. En julio de 2007 se han actualizado las estimaciones de 2002 a 2007. Los datos de 2006 y 2007 son una estimación provisional y los de 2008 son una estimación avance.

Fuente: Elaboración propia a partir de datos del INE (2010)

Como puede constatarse, el crecimiento interanual del Producto Interior Bruto (PIB) por habitante en Melilla ha estado por encima del total de España entre 2005 y 2009, a excepción de 2007 y con la particularidad de que en 2008 el diferencial a favor de Melilla ha sido más escaso (0’4 puntos porcentuales) que en los dos primeros años del período (2'1 y 1'8 puntos porcentuales respectivamente). En términos de crecimiento acumulado en el citado período, siempre ha existido un diferencial favorable y progresivo a favor de Melilla; ha pasado de 2'1 puntos porcentuales en 2005 a 4'4 en 2008. El 
cálculo de la correlación entre las bonificaciones de cuotas a la Seguridad Social y el importe en euros por habitante del PIB a precios de mercado, referidas ambas variables a Melilla, da un elevadísimo Coeficiente de Correlación: 0,977630 (con un Coeficiente R2 de 0,955760 ). El problema es determinar cuál de ambas variables se comporta como independiente y cuál como dependiente, así como efectuar las mediciones precisas. En principio, sería el crecimiento económico el que explicaría el aumento del número de trabajadores concernidos por las bonificaciones y del importe de éstas (en un marco estable de sectores afectados y del 40\% de bonificación); sin embargo, también las propias bonificaciones impulsarían, a través del crecimiento del empleo, el crecimiento económico. Estaríamos entonces ante correlaciones cruzadas que precisarían de un análisis para que por el momento no disponemos de los datos necesarios.

Al objeto de intentar pasar ahora a un análisis con el mayor grado de desagregación que nos permitan los datos disponibles, se insertan a continuación la tabla (3.12) y el gráfico (3.14), elaborados a partir de la información disponible sobre la evolución del Valor Añadido Bruto (VAB) a precios básicos por sectores de actividad, para Melilla y el total de España en el período 2004-2008

Pueden observarse las siguientes características de la estructura sectorial de Melilla: a) La agricultura, ganadería y pesca continúa siendo prácticamente testimonial, sin duda por la carencia de recursos naturales; ${ }^{18}$ b) La industria tiene una participación muy por debajo de la media del total de España, evolucionando a lo largo del período estudiado entre el 4'6\% y el 4’4\% (España entre el 18'5\% y el 17’0\%); c) La construcción presenta un peso relativo similar al del conjunto de España, siempre dentro de la evolución descendente general iniciada en 2007; d) Los servicios presentan en el caso de Melilla un peso mayor que en el conjunto de España: en 2008 el 85’ 4\% en Melilla (frente al 69'0\% en España). Sin embargo, debe destacarse la coexistencia, dentro de este último diferencial positivo, de situaciones muy dispares; como ejemplo: mientras la Administración pública emplea al 27’85\% de los afiliados al Régimen General de la Seguridad Social, el siguiente subsector por orden de importancia: el "Comercio al por menor, excepto de vehículos de motor y motocicletas”, emplea tan sólo al 9’23\%.

${ }^{18}$ Así como, entre otras causas, por la no-pertenencia de Melilla al Territorio Aduanero Comunitario y las inocuas reglas de origen correspondientes. En tanto no cambie, en su caso, el marco citado puede considerarse una actividad sin futuro. 
Tabla 3.12: VAB

\begin{tabular}{|l|r|r|r|r|r|}
\hline \multicolumn{7}{|c|}{ VALOR AÑADIDO BRUTO } \\
\hline \multicolumn{1}{|c|}{ Distribución porcentual del VAB a precios básicos por sectores de actividad (2) } \\
\hline & 2004 & 2005 & 2006 & 2007 & 2008 \\
\hline ESPAÑA Agricultura, ganadería y pesca & 3,6 & 3,2 & 2,8 & 2,7 & 2,6 \\
\hline MELILLA Agricultura, ganadería y pesca & 0,9 & 0,8 & 0,7 & 0,7 & 0,6 \\
\hline ESPAÑA Industria & 18,5 & 18,2 & 17,8 & 17,3 & 17,0 \\
\hline MELILLA Industria & 4,6 & 4,7 & 4,5 & 4,5 & 4,4 \\
\hline ESPAÑA Construcción & 10,6 & 11,5 & 12,1 & 11,8 & 11,4 \\
\hline MELILLA Construcción & 9,2 & 9,5 & 10,1 & 9,7 & 9,5 \\
\hline ESPAÑA Servicios & 67,3 & 67,1 & 67,4 & 68,1 & 69,0 \\
\hline MELILLA Servicios & 85,2 & 85,0 & 84,7 & 85,1 & 85,4 \\
\hline
\end{tabular}

Gráfico 3.14: VAB
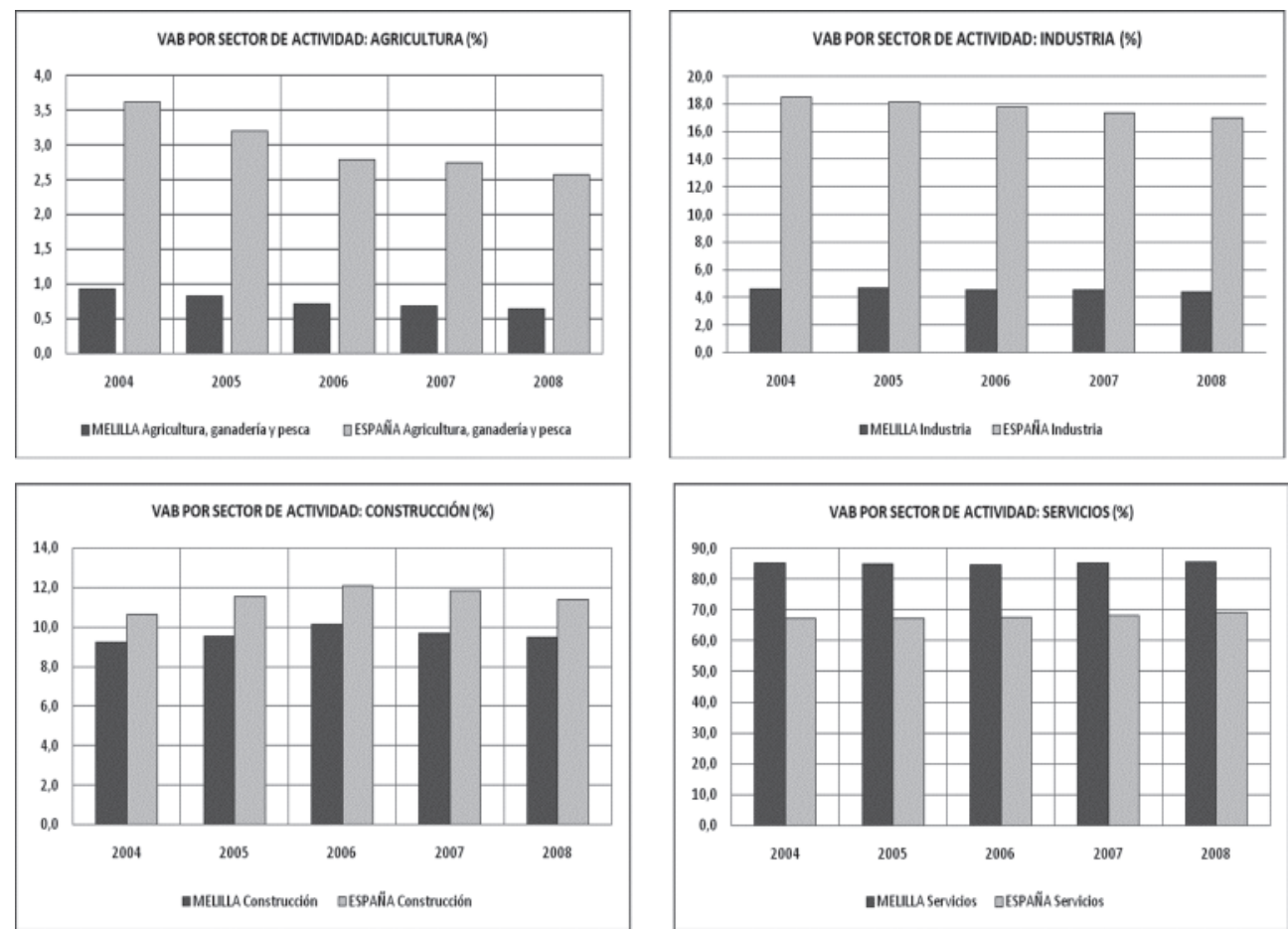

(2) La industria no incluye la construcción. Datos 2006 y 2007: estimación provisional; 2008: estimación avance.

Fuente: Elaboración propia a partir de datos del INE (2010) 


\section{CONCLUSIONES}

Las conclusiones que cabe extraer para el caso de la ciudad de Melilla, sobre la base del trabajo desarrollado, son las siguientes:

\section{$1^{\text {a }}$. Afiliación a la Seguridad Social:}

El análisis de la evolución en el período 2004-2010 justifica que, si se quieren reactivar los efectos positivos registrados hasta 2009 sobre la afiliación total a la Seguridad Social en Melilla, se adopten medidas adicionales a las iniciadas en 2004 en materia de bonificación de cuotas, bien entendido que la bonificación no es la única causa explicativa de los efectos finales sobre la citada afiliación.

\section{$2^{a}$. Bonificaciones:}

Resulta válido también aquí, mutatis mutandis, lo ya dicho en el epígrafe anterior. Es decir, que la incapacidad a la que se ha llegado para permitir efectos adicionales a los conseguidos hasta ahora, justificaría asimismo, si se quieren impulsar los objetivos sociales sobre Melilla buscados por el sistema de bonificación de cuotas a la Seguridad Social, la necesidad de adoptar nuevas medidas.

\section{$3^{\text {a }}$. Contrataciones:}

Resulta asimismo válida en este ámbito, también mutatis mutandis, la conclusión obtenida en el caso del primero. En efecto, la evolución de la situación y su deterioro a partir de 2007 justifican, si se quiere reactivar los efectos positivos sobre la contratación de trabajadores en Melilla, la necesidad de adoptar medidas adicionales a las iniciadas en 2004 en materia de bonificación de cuotas, bien entendido también que la bonificación no es la única causa explicativa de los efectos finales sobre la citada contratación.

\section{$4^{\mathrm{a}}$. Tasas de actividad:}

La moderada correlación positiva existente entre el aumento de bonificaciones en Melilla y la evolución del número de personas activas, permite concluir que una ampliación y extensión de las bonificaciones tendrá algún efecto, positivo y moderado asimismo, en esta evolución. Las implicaciones a tener presente en tal escenario son dos: por una parte, las bonificaciones trabajan a favor del crecimiento económico al permitir incrementar la población activa con relación 
a la inactiva (siempre que exista una repercusión positiva en el empleo); y por otro lado, dicho incremento de la población activa ejerce un efecto indeterminado en términos de tasa de paro, según sea su composición entre parados y ocupados. ${ }^{19}$

\section{5a. Tasas de empleo:}

La tímida convergencia de la tasa de empleo de Melilla respecto de la de España, experimentada recientemente, coexiste, significativamente, con un descenso de esta última. En consecuencia, las bonificaciones actualmente vigentes no pueden considerarse con entidad suficiente para consolidar una nueva etapa de correlación positiva con la tasa de empleo. Por otra parte, la concurrencia de una baja correlación no debe entenderse en el sentido de que deba prescindirse de la variable bonificaciones, ya que podría ser válido el acompañarse de otras de manera que la combinación de todas ellas permitiera efectos sinérgicos. En el mejor de los casos, las bonificaciones serían, en sus configuraciones actuales, necesarias pero no suficientes. Además, no deben obviarse las prevenciones a introducir en el análisis por causa de los conocidos planes de empleo.

\section{6a. Paro:}

Si bien es cierto que no puede documentarse una correlación negativa entre las bonificaciones en Melilla y la evolución de la tasa de paro, no lo es menos que, al encontrarnos en el ámbito de actuación de fenómenos sociales (y por ello existir restricciones insuperables para realizar determinadas experimentaciones ancladas en la realidad), no puede descartarse a priori que, sin la concurrencia de las citadas bonificaciones, la tasa de paro pudiera haber evolucionado de manera aún peor que la finalmente registrada; en tal supuesto, sí existiría correlación negativa entre las bonificaciones y la citada tasa. De hecho, la tasa de paro de Melilla y España según la EPA, ${ }^{20}$ han acabado convergiendo prácticamente en el primer trimestre de 2010, si bien sólo en términos totales ${ }^{21}$ (persisten importantes diferenciales en contra de Melilla en el caso de los

${ }^{19}$ Recuérdese:

${ }^{20}$ El SPEE, antiguo INEM, hace tiempo que dejó de publicarla.

${ }^{21}$ Sin perjuicio de lo ya recordado en una nota anterior, acerca de que la tasa de Melilla debe tomarse con precaución debido a los problemas de muestreo de quepuede adolecer. 
menores de 25 años y de las mujeres). Adicionalmente, y como ya se ha dicho en la conclusión anterior, deben también tenerse presentes en este caso las prevenciones a introducir en el análisis como consecuencia de los conocidos planes de empleo.

\section{$7^{\mathrm{a}}$. Convenios colectivos:}

Si bien es asimismo cierto que las bonificaciones no se han traducido en que los trabajadores hayan tenido una mayor cobertura de convenios colectivos, no lo es menos que el porcentaje de aumento salarial revisado sí ha crecido, encontrando una explicación en términos de correlación, si bien limitada, en las bonificaciones vigentes desde 2004.

\section{$8^{\text {a }}$. Remuneraciones por asalariado:}

El comportamiento en Melilla de las remuneraciones de los asalariados permite concluir: a) Se ha registrado un descenso en el caso de dos ramas de actividad bonificadas para las que se dispone de datos específicos: la industria (-7'1\%) y la hostelería (-11'4\%), por lo que al menos en estos dos casos no puede documentarse que las bonificaciones hayan tenido una repercusión positiva sobre las remuneraciones de los asalariados; b) Si bien en términos agregados sectoriales se ha producido un aumento y, además, este ha sobrepasado el registrado en el conjunto de España, habida cuenta de lo acabado de expresar para el caso de industria y hostelería no puede documentarse que tal aumento haya sido debido a las bonificaciones, salvo en el caso de que hayan concurrido lo que se conoce como subvenciones (o ayudas, bonificaciones, etc.) empresariales cruzadas; c) La no-detección del hipotético impacto que las bonificaciones habrían originado sobre las remuneraciones de los asalariados, debe entenderse sin perjuicio de la existencia de efectos, en su caso, sobre otras variables.

\section{$9^{a}$. Contratación indefinida:}

Considerando la existencia de dos etapas diferenciadas dentro del período analizado: en este caso 2004-2008, se comprueba que las mismas son coherentes con lo observado al respecto en el caso de otras variables antes examinadas. En efecto, las bonificaciones parecen haber constituido un revulsivo para avanzar en el logro de determinados objetivos sociales, en este caso y de manera limitada 
la contratación indefinida, pero tras unos primeros años de efectos positivos el modelo se muestra incapaz de generar efectos añadidos, por lo que habría llegado el momento de adoptar medidas adicionales.

\section{$10^{a}$.VAB por habitante:}

La estructura económica de Melilla sigue presentando graves desequilibrios sectoriales. Sus características más destacables son: un sector industrial cuya participación en el total delVAB está muy por debajo del caso del total nacional; y un sector servicios donde, junto a la excesiva dependencia del mismo que supone su elevada participación en el total del VAB, coexisten subsectores con unos porcentajes o muy por debajo, o muy por encima del caso de España en su conjunto.

\section{CONSIDERACIONES FINALES}

Finalmente, las observaciones que el autor del presente trabajo considera pertinentes, a partir de las conclusiones alcanzadas, son las siguientes:

$1^{\text {a }}$. El análisis de la evolución en el período 2004-2010 justifica que, si se quieren reactivar los efectos positivos registrados hasta 2009 sobre la afiliación total a la Seguridad Social en Melilla, se adopten medidas adicionales a las iniciadas en 2004 en materia de bonificación de cuotas, bien entendido que la bonificación no es la única causa explicativa de los efectos finales sobre la citada afiliación.

$2^{\text {a }}$. Parece fuera de toda duda razonable que la extensión y ampliación de las actuales bonificaciones de determinadas cuotas de la Seguridad Social en la ciudad de Melilla, resultan efectivas para persistir en el logro de los objetivos sociales pretendidos por esta medida desde su puesta en marcha en 2004; en cuanto a su eficiencia, no han sido detectadas hasta ahora medidas alternativas contrastadas como mejores. La extensión y la ampliación citadas no tienen por qué ser conceptos unívocos; en efecto, lo acabado de concluir sobre ambas medidas es igualmente aplicable tanto a extensiones sectoriales que presenten determinadas excepciones, como a ampliaciones porcentuales moduladas diferencialmente.

$3^{a}$. Otra cuestión distinta es que, si bien dichas medidas se muestran necesarias, no existe certeza sobre su suficiencia para la consecución satisfactoria de los 
objetivos buscados. Pero ello no justifica que las mismas deban eliminarse. Sensu contrario, deben complementarse con otras actuaciones adicionales que procuren, al menos, los mismos objetivos sociales que en 2003 (esencialmente la generación de empleo y su mantenimiento), hoy más difíciles de conseguir en el contexto de una crisis global que, sobrevenida posteriormente, aún persiste.

$4^{\mathrm{a}}$. En línea con lo sostenido por algunos de los diputados que anunciaron en sus intervenciones su voto a favor o su abstención, la aceptación de la toma en consideración de la proposición de ley de la Asamblea de Melilla, no tenía por qué ser incompatible con su perfeccionamiento en el curso del debate ulterior y, consecuentemente, con su aprobación final en los términos que, en su caso, se consensuaran.

$5^{a}$. En consecuencia, el rechazo por el Congreso de los Diputados a la toma en consideración de la proposición de ley de la Asamblea de Melilla, relativa a la extensión y ampliación de las bonificaciones de determinadas cuotas a la Seguridad Social, supone una ocasión perdida para posibilitar que dicha ciudad avance significativamente en el alcance de los objetivos sociales básicos que su Asamblea demanda.

6 . En el corto o medio plazo queda una nueva oportunidad, en tanto la Ciudad Autónoma de Ceuta ha dejado pendiente la defensa, también en el Congreso de los Diputados, de otra proposición de ley similar a la defendida sin éxito por la Ciudad Autónoma de Melilla y que asimismo afectaría a ambas ciudades.

$7^{a}$. Por último, debe señalarse que la evolución reciente de las magnitudes macroeconómicas de España, y en particular de las presupuestarias, introduce nuevos elementos cuya repercusión sobre el sistema aquí analizado parece negativa. Pero ese interrogante constituye el objeto de otro análisis que, recurriendo a Max Weber en El Político y el Científico, parece oportuno que comenzara por dilucidar si debe desarrollarse bajo la ética de la convicción propia del científico o bajo la ética de la responsabilidad propia del político.

\section{NOTAS (NORMATIVA BÁSICA CONSULTADA)}

ESPAÑA. MINISTERIO DETRABAJO E INMIGRACIÓN. Orden TIN/530/2010, de 5 de marzo, por la que se prorroga la aplicación de las bonificaciones de cuotas regulada en la 
Orden TAS/471 / 2004, de 26 de febrero, por la que se dictan normas para la aplicación de las bonificaciones de cuotas establecidas por el apartado 2 de la disposición adicional trigésima del texto refundido de la Ley General de la Seguridad Social, respecto de empresas y trabajadores por cuenta propia de las Ciudades de Ceuta y Melilla y se modifica dicha orden. BOE No 59 de 9/3/2010, p. 23637-23638.

ESPAÑA. MINISTERIO DETRABAJOY ASUNTOS SOCIALES. Orden TAS/710/2008, de 7 de marzo, por la que se prorroga la aplicación de las bonificaciones de cuotas regulada en la Orden TAS / 471 / 2004, de 26 de febrero, por la que se dictan normas para la aplicación de las bonificaciones de cuotas establecidas por el apartado 2 de la disposición adicional trigésima del Texto Refundido de la Ley General de la Seguridad Social, respecto de empresas y trabajadores por cuenta propia de las Ciudades de Ceuta y Melilla y se modifica dicha Orden. BOE N 66 de 17/3/2008, p. 15870-15871.

ESPAÑA. MINISTERIO DETRABAJOY ASUNTOS SOCIALES. Orden TAS/856/2006, de 21 de marzo, por la que se prorroga la aplicación de las bonificaciones de cuotas reguladas en la Orden TAS /471 / 2004, de 26 de febrero, por la que se dictan normas para la aplicación de las bonificaciones de cuotas establecidas por el apartado 2 de la disposición adicional trigésima del Texto Refundido de la Ley General de la Seguridad Social, respecto de empresas y trabajadores por cuenta propia de las Ciudades de Ceuta y Melilla y se modifica dicha Orden. BOE N ${ }^{\circ} 74$ de 28/3/2006, p. 11813.

ESPAÑA. MINISTERIO DETRABAJOY ASUNTOS SOCIALES. Orden TAS/471/2004, de 26 de febrero, por la que se dictan normas para la aplicación de las bonificaciones de cuotas establecidas por el apartado 2 de la disposición adicional trigésima del Texto Refundido de la Ley General de la Seguridad Social, respecto de empresas y trabajadores por cuenta propia de las ciudades de Ceuta y Melilla. BOE No 9236 de 27/2/2004, p. 92369237.

ESPAÑA. MINISTERIO DE TRABAJOY SEGURIDAD SOCIAL. Real Decreto legislativo 1/1994, de 20 de junio, por el que se aprueba el texto refundido de la Ley General de la Seguridad Social. BOE No 154 de 29/6/1994, p. 20658-20708 [Con modificaciones posteriores].

\section{BIBLIOGRAFÍA}

BENTOLILA, S.; SEGURA SÁNCHEZ, J.; TOHARIA CORTÉS, L. «La contratación temporal en España: un análisis económico». Moneda y Crédito. 1991. 
BENTOLILA, S.; TOHARIA CORTÉS, L. Estudios de economía del trabajo en España, III: el problema del paro. Madrid: Ministerio de Trabajo y Seguridad Social, Colección Economía y Sociología del Trabajo [Comps.], 1991.

CEBRIÁN LÓPEZ, I; GARRIDO MEDINA, L.J.; TOHARIA CORTÉS, L. «El paro de larga duración en España». El problema del paro de larga duración en España. Madrid: INEM, 1992.

$\longrightarrow$ Los parados de larga duración y la protección social». Política social y Estado del bienestar [PÉREZYRUELA, M; MORENO, I.]. Madrid: Ministerio de Asuntos Sociales, Colección Estudios, 1991.

DELORS, J. Véase: UNIÓN EUROPEA. COMISIÓN DE LAS COMUNIDADES EUROPEAS.

ESPAÑA. AGENCIA ESTATAL DE EVALUACIÓN DE LAS POLÍTICAS PÚBLICASY LA CALIDAD DE LOS SERVICIOS. La bonificación de las cuotas sociales a la Seguridad Social en Ceuta y Melilla para estimular la creación de empleo. Madrid: Ministerio de Administraciones Públicas, 2008, Catálogo general de publicaciones oficiales: NIPO: 331-08-006-8.

ESPAÑA. CORTES GENERALES. CONGRESO DE LOS DIPUTADOS. Toma en consideración de proposiciones de ley de comunidades autónomas: De la Asamblea de la Ciudad Autónoma de Melilla, para la modificación de la disposición adicional trigésima del real decreto legislativo 1/1994, de 20 de junio, por el que se aprueba el texto refundido de la ley general de la seguridad social. Boletín Oficial de las Cortes Generales. Congreso de los Diputados, 11 de mayo de 2010 [ $\left.\mathrm{N}^{\circ} 161\right]$.

Informe de evaluación de los resultados del actual sistema de bonificaciones a la contratación. [Trabajo e Inmigración]. Boletín Oficial de las Cortes Generales. Congreso de los Diputados, 17 de marzo de 2010 [ $\mathrm{N}^{\circ}$ 496].

Informe de la Ponencia para el análisis de los problemas estructurales del sistema de la Seguridad Social y de las principales reformas que deberán acometerse. [Más conocido como "Pacto deToledo"] [Título IX: Recomendaciones]. Boletín Oficial de las Cortes Generales. Congreso de los Diputados, 12 de abril de 1996 [ $\left.N^{\circ} 134\right]$.

FINA SANGLAS. LI. «El paro en España: sus causas y las respuesta de la política económica». Las causas del paro en España: un punto de Vista estructural [FINA, Ll; TOHARIA, L.]. Madrid: Fundación, 1987.

JIMENO SERRANO, J.F.; TOHARIA CORTÉS, L. «El mercado de trabajo: lo que hay que reformar y por qué». Economistas [No 57, P- 13-21]. Madrid: CEM, 1993, ISSN 0212-4386. 
"The effects of fixed-term employment on wages: theory and evidence from Spain". Investigaciones económicas [Vol. 17, No 3, p. 475-494]. Madrid: 1993.

Unemployment and labour market flexibility - Spain. Ginebra: OIT, 1992.

"El mercado de trabajo español en el proceso de convergencia hacia la unión económica y monetaria europea”. Papeles de economía española. Madrid [ $\mathrm{N}^{\circ}$ 52-53, p. 78-107], 1992, ISSN 0210-9107.

"The productivity and wage effects of fixed-term contracts: evidence from Spain". Tercera Conferencia Anual de la Asociación Europea de Economistas del Trabajo [Ponencia]. El Escorial: septiembre, 1991.

MOLINA NAVARRETE, C. Estrategias de competitividad, mercados de trabajo y reforma laboral 2010: convergencias y divergencias. Madrid: Centro de Estudios Financieros, 2011, ISBN: 9788445416327.

SEGURA SÁNCHEZ, J. et al. Análisis de la contratación temporal en España. Madrid: Ministerio de Trabajo y Seguridad Social, Colección Economía y Sociología del Trabajo, 1991.

TOHARIA CORTÉS, L. La temporalidad en el empleo: atrapamiento y trayectorias. Madrid: Ministerio de Asuntos Sociales, 2007; ISBN: 9788484172437.

__ "El mercado de trabajo en España: situación y perspectivas". Revista económica de Castilla - La Mancha [No 4, p. 77-106]. Toledo: Clm. Economía, 2004.

_ El mercado de trabajo: teorías y aplicaciones. Madrid: Alianza Editorial - Colección Alianza Universidad, 1983, ISBN: 9788420680705.

TOHARIA CORTÉS, L.; MALO OCAÑA, M.A. «¿Qué se puede esperar de las reformas del mercado de trabajo?». Circunstancia: revista de ciencias sociales del Instituto Universitario de Investigación Ortega y Gasset [ $\mathrm{N}^{\circ}$ 20, Ejemplar dedicado a «La reforma laboral en España: propuestas para el debate»]. Madrid: IUIOG, 2009, ISSN 16961277.

UNIÓN EUROPEA. COMISIÓN DE LAS COMUNIDADES EUROPEAS. 'Libro Blanco: crecimiento, competitividad, empleo, retos y pistas para entrar en el siglo XXI. ' [Conocido como 'Libro blanco de Delors]. Luxemburgo: Oficina de Publicaciones Oficiales, 1993 [diciembre]. 